\title{
Thermalization of the mildly relativistic plasma
}

\author{
A.G. Aksenov \\ Institute for Theoretical and Experimental Physics, \\ B. Cheremushkinskaya 25, 117218 Moscow, \\ Russia and Institute for Computer-Aided Design, Russian Academy of Sciences, \\ Vtoraya Brestskaya 19/18, Moscow, 123056, Russia \\ R. Ruffini and G.V. Vereshchagin \\ ICRANet p.le della Repubblica, 10, 65100 Pescara, Italy and \\ ICRA and University of Rome "Sapienza", \\ Physics Department, p.le A. Moro 5, 00185 Rome, Italy
}

\begin{abstract}
In the recent Letter [1] we considered the approach of nonequilibrium pair plasma towards thermal equilibrium state adopting a kinetic treatment and solving numerically the relativistic Boltzmann equations. It was shown that plasma in the energy range $0.1-10 \mathrm{MeV}$ first reaches kinetic equilibrium, on a timescale $t_{\mathrm{k}} \lesssim 10^{-14} \mathrm{sec}$, with detailed balance between binary interactions such as Compton, Bhabha and Møller scattering, and pair production and annihilation. Later the electron-positron-photon plasma approaches thermal equilibrium on a timescale $t_{\mathrm{th}} \lesssim 10^{-12}$ sec, with detailed balance for all direct and inverse reactions. In the present paper we systematically present details of the computational scheme used in [1], as well as generalize our treatment, considering proton loading of the pair plasma. When proton loading is large, protons thermalize first by proton-proton scattering, and then with the electron-positron-photon plasma by proton-electron scattering. In the opposite case of small proton loading proton-electron scattering dominates over proton-proton one. Thus in all cases the plasma, even with proton admixture, reaches thermal equilibrium configuration on a timescale $t_{\text {th }} \lesssim 10^{-11}$ sec. We show that it is crucial to account for not only binary but also triple direct and inverse interactions between electrons, positrons, photons and protons. Several explicit examples are given and the corresponding timescales for reaching kinetic and thermal equilibria are determined.
\end{abstract}




\section{INTRODUCTION}

An electron-positron plasma is of interest in many fields of physics and astrophysics. One of the crucial quantities in this analysis is the timescale of the thermalization process. In the early universe [2],[3],[4],[5] during the lepton era, ultrarelativistic electron-positron pairs contribute to the matter contents of the Universe. In gamma-ray bursts (GRBs) electronpositron pairs play essential role in the dynamics of expansion [6], [7], [8]. Indications exist on the presence of the pair plasma also in active galactic nuclei [9], in the center of our Galaxy [10], around hypothetical quark stars [11]. In the laboratory pair plasma is expected to appear in the fields of ultra intense lasers [12], where particle production may serve as a diagnostic tool for high-energy plasma [13].

In many stationary astrophysical sources the pair plasma is thought to be in thermodynamic equilibrium. A detailed study of the relevant processes [14], [15], [16], [17], [18], [19], radiatiation mechanisms [20], possible equilibrium configurations [16],[21], [22] and spectra [23] in an optically thin pair plasma has been carried out. Particular attention has been given to collisional relaxation process [24],[25], pair production and annihilation [26], relativistic bremsstrahlung [27], [28], double Compton scattering [29], [30].

An equilibrium occurs if the sum of all reaction rates vanishes. For instance, electronpositron pairs are in equilibrium when the net pair production (annihilation) rate is zero. This can be achieved by variety of ways and the corresponding condition can be represented as a system of algebraic equations [31]. However, the main assumption made in all the above mentioned works is that the plasma is assumed to obey relativistic quantum statistics. The latter is shown to be possible, in principle, in the range of temperatures up to $10 \mathrm{MeV}$ [14],[25]. Our main task is to prove that independently of a wide set of initial conditions, thermal equilibruim forms for the phase space distribution functions are recovered during the process of thermalization by two body and three body direct and inverse particle-particle collisions.

At the same time, in some cases mentioned above the pair plasma can be optically thick. Although moderately thick plasmas have been considered in the literature [22], only qualitative description [14],[21] is available for large optical depths. Assumption of thermal equilibrium is often adopted for rapidly evolving systems such as GRBs without explicit proof [6], [7], [8], [32]. Then hydrodynamic approximation is usually applied both for leptons 
and photons. However, particles may not be in equilibrium initially. Moreover, they may not reach an equilibrium in rapidly evolving systems such as the early Universe or transient events, when the energy is released on a very short timescale.

In the literature there is no consensus on this point. Some authors considered thermal equilibrium as the initial state prior to expansion [6], [8], while others did not [33]. In fact, the detailed study of the pair plasma equilibrium configurations, performed in [21], cannot answer this question, because essentially nonequilibrium processes have to be considered.

Thus, observations provide motivation for theoretical analysis of physical conditions taking place in nonequilibrium optically thick pair plasma. Notice that there is substantial difference between the ion-electron plasma on the one hand and electron-positron plasma on the other hand. Firstly, the former is collisionless in the wide range of parameters [34], while collisions are always essential in the latter. Secondly, when collisions are important relevant interactions in the former case are Coulomb scattering of particles which are usually described by the classical Rutherford cross-section. In contrast, interactions in the pair plasma are described by quantum cross-sections even if the plasma itself can be still treated as classical one.

Our study reported in [1],[35] in the case of pure pair plasma clarified the issue of initial state of the pair plasma in GRBs sources. Our numerical calculations show that the pair plasma on a timescale $t \lesssim 10^{-12}$ sec reach thermal equilibrium prior to expansion, due to intense binary and triple collisions. In this paper we present details about the computational scheme adopted in [1] and turn to a more general case, the pair plasma loaded with baryons. Occurence of the thermalization process and the corresponding timescales are necessary for determining the dynamics of GRBs. Thermalization timescales $t \lesssim 10^{-12}$ sec are indeed necessary in order to relate the observed properties of GRBs to the nature of the source, see e.g. [36].

In the next Section we give qualitative description of the pair plasma, introducing some relevant parameters. In Section 3 we discuss pure pair plasma. In Section 4 pair plasma with proton loading is discussed. In Section 5 we describe the computational scheme used in our analysis. In Section 6 we present results of numerical computations. Discussion and conclusions follow in the last Section. In Appendix A relevant conservation laws are recalled. In Appendix B conditions for kinetic and thermal equilibria are formulated, and the scheme for determination of temperatures and chemical potentials out of number and energy 
densities are given. Binary interactions in the pair plasma such as Compton, Møller and Bhabha scatterings, as well as pair creation and annihilation by two photons are discussed in Appendix C. In Appendix D Compton and Coulomb scatterings with protons are considered. In Appendix E three-body radiative variants of the reactions listed above are given. Cutoff scheme for numerical evaluation of emission and absorption coefficients are presented in Appendix F. In Appendix G mass scaling of the matrix elements for Coulomb scattering between electrons, positrons and protons is discussed. In Appendix $\mathrm{H}$ the definition of matrix elements and cross-sections adopted in the paper are given.

\section{QUALITATIVE DESCRIPTION OF THE PAIR PLASMA}

First of all we specify the domain of parameters characterizing the pair plasma considered in this paper. It is convenient to use dimensionless parameters usually adopted for this purpose.

We consider mildly relativistic pair plasma, thus the average energy per particle $\epsilon$ brackets the electron rest mass energy

$$
0.1 \lesssim \frac{\epsilon}{m c^{2}} \lesssim 10
$$

The lower boundary is required for significant concentrations of pairs, while the upper boundary is set to avoid substantial production of other particles such as muons and neutrinos.

We define the plasma parameter $\mathfrak{g}=\left(n_{-} d^{3}\right)^{-1}$, where $d=\sqrt{\frac{k_{B} T_{-}}{4 \pi e^{2} n_{-}}}=\frac{c}{\omega} \sqrt{\theta_{-}}$is the Debye length, $k_{B}$ is Boltzmann's constant, $e, n_{-}$and $T_{-}$are the electron charge, number density and temperature respectively, $c$ the is speed of light, $\theta_{-}=k_{B} T_{-} /\left(m c^{2}\right)$ is dimensionless temperature, $\omega=\sqrt{4 \pi e^{2} n_{-} / m}$ is the plasma frequency and $m$ is the electron mass. To ensure applicability of kinetic approach it is necessary that the plasma parameter is small, $\mathfrak{g} \ll 1$. This condition means that kinetic energy of particles dominates their potential energy due to mutual interaction. For the pair plasma considered in this paper this condition is satisfied.

Further, the classicality parameter, defined as $\varkappa=e^{2} /\left(\hbar v_{r}\right)=\alpha / \beta_{r}$, where $\hbar$ is Planck's constant, $\alpha=e^{2} /(\hbar c)$ is the fine structure constant, $v_{r}=\beta_{r} c$ is mean relative velocity of particles, see $(\underline{\mathrm{F} 12})$ in Appendix. The condition $\varkappa \gg 1$ means that particles collisions can be considered classically, while for $\varkappa \ll 1$ quantum description is required. In our case both for pairs and protons quantum cross-sections are used since $\varkappa<1$. 
The strength of screening of the Coulomb interactions is characterized by the Coulomb $\operatorname{logarithm} \Lambda=\mathcal{M} d v_{r} / \hbar$, where $\mathcal{M}$ is the reduced mass. For electron-electron or electronpositron scattering the reduced mass is just $m / 2$, while for electron-proton or positron-proton scattering the reduced mass is just the proton mass $\mathcal{M} \simeq M$; for proton-proton scattering $\mathcal{M} \simeq M / 2$. Coulomb logarithm varies with mean particle velocity and Debye length, and it cannot be set a constant as is usually done in most of studies of the pair plasma.

Finally, we consider pair plasma with linear dimensions $R$ exceeding the mean free path of photons $l=\left(n_{-} \sigma\right)^{-1}$, where $\sigma$ is the corresponding total cross-section. Thus the optical depth $\tau=n \sigma R \gg 1$ is large, and interactions between photons and other particles have to be taken in due account. We discuss these interaction in the next Section.

Note that natural parameters for perturbative expansion in the problem under consideration are the fine structure constant $\alpha$ and the electron-proton mass ratio $m / M$.

\section{PURE PAIR PLASMA}

For simplicity we first consider pure pair plasma composed of electrons $e^{-}$, positrons $e^{+}$, and photons $\gamma$. We will turn to a more general case, including protons $p$ in the next Section. We assume that pairs or photons appear by some physical process in the region with a size $R$ and on a timescale $t<R / c$. We further assume that distribution functions of particles depend neither on spatial coordinates nor on the direction of momenta. We then have $f_{i}=f_{i}(\epsilon, t)$, namely we consider isotropic distributions functions in momentum space for a spatially uniform and isotropic plasma.

To make sure that classical kinetic description is adequate we estimate the dimensionless degeneracy temperature

$$
\theta_{F}=\left[\left(\frac{\hbar}{m c}\right)^{2}\left(3 \pi^{2} n_{-}\right)^{\frac{2}{3}}+1\right]^{1 / 2}-1
$$

and compare it with the estimated temperature in thermal equilibrium. With our initial conditions (11) the degeneracy temperature is always smaller than the temperature in thermal equilibrium and therefore we can safely apply the classical kinetic approach. Besides, since we deal with ideal plasma with the plasma parameter $\mathfrak{g} \sim 10^{-3}$ it is enough to consider only one-particle distribution functions. These considerations justify our computational approach based on classical relativistic Boltzmann equation. At the same time the right hand side of 
Boltzmann equations contains collisional integrals as functions of quantum matrix elements, as discussed below and in Appendices C-E.

Relativistic Boltzmann equations [37],[38] in spherically symmetric case for which the original code is designed [39] are

$$
\begin{gathered}
\frac{1}{c} \frac{\partial f_{i}}{\partial t}+\beta_{i}\left(\mu \frac{\partial f_{i}}{\partial r}+\frac{1-\mu^{2}}{r} \frac{\partial f_{i}}{\partial \mu}\right)-\nabla U \frac{\partial f_{i}}{\partial \mathbf{p}}= \\
=\sum_{q}\left(\eta_{i}^{q}-\chi_{i}^{q} f_{i}\right)
\end{gathered}
$$

where $\mu=\cos \vartheta, \vartheta$ is the angle between the radius vector $\mathbf{r}$ from the origin and the particle momentum $\mathbf{p}, U$ is a potential due to an external force, $\beta_{i}=v_{i} / c$ are particles velocities, $f_{i}(\epsilon, t)$ are their distribution functions, the index $i$ denotes the type of particle, $\epsilon$ is its energy, and $\eta_{i}^{q}$ and $\chi_{i}^{q}$ are the emission and the absorption coefficients for the production of a particle of type " $i$ " via the physical process labeled by $q$. This is a coupled system of partial-integro-differential equations. For homogeneous and isotropic distribution functions of electrons, positrons and photons (3) reduces to

$$
\frac{1}{c} \frac{\partial f_{i}}{\partial t}=\sum_{q}\left(\eta_{i}^{q}-\chi_{i}^{q} f_{i}\right),
$$

which is a coupled system of integro-differential equations. In (44) we also explicitly neglected the Vlasov term, describing collisionless interaction of particles in the mean field, since energy density of fluctuations of the electromagnetic field are many orders of magnitude smaller than the energy density of particles [40].

Therefore, the left-hand side of the Boltzmann equation is reduced to partial derivative of the distribution function with respect to time. The right-hand side contains collisional integrals, representing interactions between electrons, positrons and photons.

As example of collisional integral consider absorption coefficient for Compton scattering which is given by

$$
\chi^{\mathrm{cs}} f_{\gamma}=\int d \mathbf{k}^{\prime} d \mathbf{p} d \mathbf{p}^{\prime} W_{\mathbf{k}^{\prime}, \mathbf{p}^{\prime} ; \mathbf{k}, \mathbf{p}} f_{\gamma}(\mathbf{k}, t) f_{ \pm}(\mathbf{p}, t),
$$

where $\mathbf{p}$ and $\mathbf{k}$ are momenta of electron (positron) and photon respectively, $d \mathbf{p}=$ $d \epsilon_{ \pm} d o \epsilon_{ \pm}^{2} \beta_{ \pm} / c^{3}, d \mathbf{k}^{\prime}=d \epsilon_{\gamma}^{\prime} \epsilon_{\gamma}^{\prime 2} d o_{\gamma}^{\prime} / c^{3}$ and the transition function $W_{\mathbf{k}^{\prime}, \mathbf{p}^{\prime} ; \mathbf{k}, \mathbf{p}}$ is related to the transition probability differential $d w_{\mathbf{k}^{\prime}, \mathbf{p}^{\prime} ; \mathbf{k}, \mathbf{p}}$ per unit time as

$$
W_{\mathbf{k}^{\prime}, \mathbf{p}^{\prime} ; \mathbf{k}, \mathbf{p}} d \mathbf{k}^{\prime} d \mathbf{p}^{\prime} \equiv V d w_{\mathbf{k}^{\prime}, \mathbf{p}^{\prime} ; \mathbf{k}, \mathbf{p}}
$$


The differential probability $d w_{\mathbf{k}^{\prime}, \mathbf{p}^{\prime} ; \mathbf{k}, \mathbf{p}}=w_{\mathbf{k}^{\prime}, \mathbf{p}^{\prime} ; \mathbf{k}, \mathbf{p}} d \mathbf{k}^{\prime} d \mathbf{p}^{\prime}$ is given by (C3) in Appendix C.

Given the momentum conservation one can perform one integration over $d \mathbf{p}^{\prime}$ in (5) as

$$
\int d \mathbf{p}^{\prime} \delta\left(\mathbf{k}+\mathbf{p}-\mathbf{k}^{\prime}-\mathbf{p}^{\prime}\right) \rightarrow 1
$$

but it is necessary to take into account the momentum conservation in the next integration over $d \mathbf{k}^{\prime}$, so we have

$$
\begin{gathered}
\int d \epsilon_{\gamma}^{\prime} \delta\left(\epsilon_{\gamma}+\epsilon_{ \pm}-\epsilon_{\gamma}^{\prime}-\epsilon_{ \pm}^{\prime}\right)= \\
=\int d\left(\epsilon_{\gamma}^{\prime}+\epsilon_{ \pm}^{\prime}\right) \frac{1}{\left|\partial\left(\epsilon_{\gamma}^{\prime}+\epsilon_{ \pm}^{\prime}\right) / \partial \epsilon_{\gamma}^{\prime}\right|} \delta\left(\epsilon_{\gamma}+\epsilon_{ \pm}-\epsilon_{\gamma}^{\prime}-\epsilon_{ \pm}^{\prime}\right) \rightarrow \\
\rightarrow \frac{1}{\left|\partial\left(\epsilon_{\gamma}^{\prime}+\epsilon_{ \pm}^{\prime}\right) / \partial \epsilon_{\gamma}^{\prime}\right|} \equiv J_{\mathrm{cs}},
\end{gathered}
$$

where the Jacobian of the transformation is

$$
J_{\mathrm{cs}}=\frac{1}{1-\beta_{ \pm}^{\prime} \mathbf{b}_{\gamma}^{\prime} \cdot \mathbf{b}_{ \pm}^{\prime}},
$$

and $\mathbf{b}_{i}=\mathbf{p}_{i} / p, \mathbf{b}_{i}^{\prime}=\mathbf{p}_{i}^{\prime} / p^{\prime}, \mathbf{b}_{ \pm}^{\prime}=\left(\beta_{ \pm} \epsilon_{ \pm} \mathbf{b}_{ \pm}+\epsilon_{\gamma} \mathbf{b}_{\gamma}-\epsilon_{\gamma}^{\prime} \mathbf{b}_{\gamma}^{\prime}\right) /\left(\beta_{ \pm}^{\prime} \epsilon_{ \pm}^{\prime}\right)$.

Finally, for the absorption coefficient we have

$$
\chi^{\mathrm{cs}} f_{\gamma}=-\int d o_{\gamma}^{\prime} d \mathbf{p} \frac{\epsilon_{\gamma}^{\prime}\left|M_{f i}\right|^{2} \hbar^{2} c^{2}}{16 \epsilon_{ \pm} \epsilon_{\gamma} \epsilon_{ \pm}^{\prime}} J_{\mathrm{cs}} f_{\gamma}(\mathbf{k}, t) f_{ \pm}(\mathbf{p}, t),
$$

where the matrix element here is dimensionless. This integral is evaluated numerically as described in Appendix.

For all binary interactions we use exact QED matrix elements which can be found in the standard textbooks, e.g. in [41],[42], (43], and are given in Appendix C.

In order to account for the charge screening we introduced the minimal scattering angles following [44], see Section $\mathbb{E}$ in Appendix. This allows to apply the same scheme for the computation of emission and absorption coefficients for Coulomb scattering, while many treatments in the literature use the Fokker-Planck approximation, e.g. [45].

For such a dense plasma collisional integrals in (4) should include not only binary interactions, having order $\alpha^{2}$ in Feynmann diagrams, but also triple ones, having order $\alpha^{3}$ [41]. As example for triple interactions consider relativistic bremsstrahlung

$$
e_{1}+e_{2} \leftrightarrow e_{1}^{\prime}+e_{2}^{\prime}+\gamma^{\prime}
$$


For the time derivative, for instance, of the distribution function $f_{2}$ in the direct and in the inverse reactions (11) one has

$$
\begin{gathered}
\dot{f}_{2}=\int d \mathbf{p}_{1} d \mathbf{p}_{1}^{\prime} d \mathbf{p}_{2}^{\prime} d \mathbf{k}^{\prime}\left[W_{\mathbf{p}_{1}^{\prime}, \mathbf{p}_{2}^{\prime}, \mathbf{k}^{\prime} ; \mathbf{p}_{1}, \mathbf{p}_{2}} f_{1}^{\prime} f_{2}^{\prime} f_{k}^{\prime}-\right. \\
\left.-W_{\mathbf{p}_{1}, \mathbf{p}_{2} ; \mathbf{p}_{1}^{\prime}, \mathbf{p}_{2}^{\prime}, \mathbf{k}^{\prime}} f_{1} f_{2}\right]=\int d \mathbf{p}_{1} d \mathbf{p}_{1}^{\prime} d \mathbf{p}_{2}^{\prime} d \mathbf{k}^{\prime} \frac{c^{6} \hbar^{3}}{(2 \pi)^{2}} \times \\
\times \frac{\delta^{(4)}\left(P_{f}-P_{i}\right)\left|M_{f i}\right|^{2}}{2^{5} \epsilon_{1} \epsilon_{2} \epsilon_{1}^{\prime} \epsilon_{2}^{\prime} \epsilon_{\gamma}^{\prime}}\left[f_{1}^{\prime} f_{2}^{\prime} f_{k}^{\prime}-\frac{1}{(2 \pi \hbar)^{3}} f_{1} f_{2}\right]
\end{gathered}
$$

where

$$
\begin{aligned}
& d \mathbf{p}_{1} d \mathbf{p}_{2} W_{\mathbf{p}_{1}^{\prime}, \mathbf{p}_{2}^{\prime}, \mathbf{k}^{\prime} ; \mathbf{p}_{1}, \mathbf{p}_{2}} \equiv V^{2} d w_{1}, \\
& d \mathbf{p}_{1}^{\prime} d \mathbf{p}_{2}^{\prime} d \mathbf{k}^{\prime} W_{\mathbf{p}_{1}, \mathbf{p}_{2} ; \mathbf{p}_{1}^{\prime}, \mathbf{p}_{2}^{\prime}, \mathbf{k}^{\prime}} \equiv V d w_{2},
\end{aligned}
$$

and $d w_{1}$ and $d w_{2}$ are given by (편) for the inverse and direct process (11) respectively. The

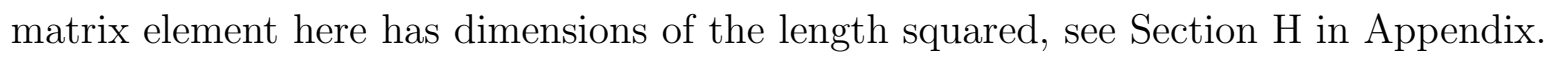

In the case of the distribution functions (15), see below, we have multipliers proportional to

$$
F_{i}=\exp \frac{\nu_{i}}{\theta_{i}},
$$

called fugacities, in front of the integrals. The calculation of emission and absorption coefficients is then reduced to the well known thermal equilibrium case [31]. In fact, since reaction rates of triple interactions are $\alpha$ times smaller than binary reaction rates, we expect that binary reactions come to detailed balance first. Only when binary reactions are all balanced, triple interactions become important. In addition, when binary reactions come into balance, distribution functions already acquire the form (15). Although there is no principle difficulty in computations using exact matrix elements for triple reactions as well, our simplified scheme allows for much faster numerical computation. The corresponding reaction rates for triple interactions are given is Section $\mathrm{E}$ in Appendix.

We consider all possible binary and triple interactions between electrons, positrons and photons as summarized in table I.

Each of the above mentioned reactions is characterized by the corresponding timescale and optical depth. For Compton scattering of an electron, for instance, we have

$$
t_{\mathrm{cs}}=\frac{1}{\sigma_{T} n_{ \pm} c}, \quad \tau_{\mathrm{cs}}=\sigma_{T} n_{ \pm} R,
$$




\begin{tabular}{|c|c|}
\hline \hline Binary interactions & $\begin{array}{c}\text { Radiative and } \\
\text { pair producing variants }\end{array}$ \\
\hline \hline Møller and Bhabha & Bremsstrahlung \\
$e_{1}^{ \pm} e_{2}^{ \pm} \longrightarrow e_{1}^{ \pm \prime} e_{2}^{ \pm \prime}$ & $e_{1}^{ \pm} e_{2}^{ \pm} \leftrightarrow e_{1}^{ \pm \prime} e_{2}^{ \pm \prime} \gamma$ \\
$e^{ \pm} e^{\mp} \longrightarrow e^{ \pm \prime} e^{\mp \prime}$ & $e^{ \pm} e^{\mp} \leftrightarrow e^{ \pm \prime} e^{\mp \prime} \gamma$ \\
\hline Single Compton & Double Compton \\
$e^{ \pm} \gamma \longrightarrow e^{ \pm} \gamma^{\prime}$ & $e^{ \pm} \gamma \leftrightarrow e^{ \pm \prime} \gamma^{\prime} \gamma^{\prime \prime}$ \\
\hline Pair production & Radiative pair production \\
and annihilation & and 3 -photon annihilation \\
$\gamma \gamma^{\prime} \leftrightarrow e^{ \pm} e^{\mp}$ & $\gamma \gamma^{\prime} \leftrightarrow e^{ \pm} e^{\mp} \gamma^{\prime \prime}$ \\
& $e^{ \pm} e^{\mp} \leftrightarrow \gamma \gamma^{\prime} \gamma^{\prime \prime}$ \\
\hline \hline
\end{tabular}

TABLE I: Microphysical processes in the pair plasma.

where $\sigma_{T}=\frac{8 \pi}{3} \alpha^{2}\left(\frac{\hbar}{m c}\right)^{2}$ is the Thomson cross-section. There are two timescales in our problem that characterize the condition of detailed balance between direct and inverse reactions, $t_{\mathrm{cs}}$ for binary and $\alpha^{-1} t_{\mathrm{cs}}$ for triple interactions respectively.

We choose arbitrary initial distribution functions and find a common development. At a certain time $t_{\mathrm{k}}$ the distribution functions always have evolved in a functional form on the entire energy range, and depend only on two parameters. We find in fact for the distribution functions the expressions

$$
f_{i}(\varepsilon)=\frac{2}{(2 \pi \hbar)^{3}} \exp \left(-\frac{\varepsilon-\nu_{i}}{\theta_{i}}\right),
$$

with chemical potential $\nu_{i} \equiv \frac{\varphi_{i}}{m c^{2}}$ and temperature $\theta_{i} \equiv \frac{k_{B} T_{i}}{m_{e} c^{2}}$, where $\varepsilon \equiv \frac{\epsilon}{m_{e} c^{2}}$ is the energy of the particle. Such a configuration corresponds to a kinetic equilibrium [3], [45], [46] in which particles acquire a common temperature and nonzero chemical potentials. At the same time we found that triple interactions become essential for $t>t_{\mathrm{k}}$, after the establishment of kinetic equilibrium. In strict mathematical sense the sufficient condition for reaching thermal equilibrium is when all direct reactions are exactly balanced with their inverse. Therefore, in principle, not only triple, but also four-particle, five-particle and so on reaction have to be accounted for in equation (4). The timescale for reaching thermal equilibrium will be then determined by the slowest reaction which is not balanced with its inverse. We stress, 
however, that the necessary condition is the detailed balance at least in triple interactions, since binary reactions do not change chemical potentials.

Notice that a method similar to ours was applied in [45] in order to compute spectra of particles in kinetic equilibrium. However, although the approach was similar, the computation was never carried out in order to actually observe the reaching of thermal equilibrium.

Finally, it is worth mentioning the physical meaning of the chemical potential $\nu_{\mathrm{k}}$ in kinetic equilibrium entering the formula (15). In the case of pure pair plasma a non-zero chemical potential represents deviation from the thermal equilibrium through the relation

$$
\nu_{\mathrm{k}}=\theta \ln \left(n_{\mathrm{k}} / n_{\mathrm{th}}\right),
$$

where $n_{\text {th }}$ are concentrations of particles in thermal equilibrium.

\section{PROTON LOADING}

So far we dealt with leptons, having the same mass but opposite charges. In that case the condition of electric neutrality is identically fulfilled. We described electrons and positrons with the same distribution function. Situation becomes more complicated when admixture of protons is allowed. Since charge neutrality

$$
n_{-}=n_{+}+n_{p}
$$

is required, the number of electrons is not equal to the number of protons. In such a case a new dimensionless parameter, the baryonic loading $\mathbf{B}$, can be introduced as

$$
\mathbf{B}=\frac{N M c^{2}}{\mathcal{E}}=\frac{n_{p} M c^{2}}{\rho_{r}}
$$

where $N$ and $n_{p}$ are the number and the concentration of protons, $\mathcal{E}$ and $\rho_{r}=\rho_{\gamma}+\rho_{+}+\rho_{-}$are radiative energy and energy density respectively. Since in relativistic plasma electrons and positrons move with almost the speed of light, both photons and pairs in thermal equilibrium

behave as relativistic fluid with equation of state $p_{r} \simeq \rho_{r} / 3$. At the same time, protons are relatively particles in the energy range (11), with negligible pressure and dust-like equation of state $p \simeq 0$. In this way by introducing parameter $\mathbf{B}$ we distinguish a radiation-dominated $(\mathbf{B}<1)$ from a matter-dominated $(\mathbf{B}>1)$ plasma. For electrically neutral plasmas there exists an upper limit on the parameter $\mathbf{B}$ defined by (18), which is $\mathbf{B} \leq M / m$. 


\begin{tabular}{|c|c|}
\hline \hline Binary interactions & $\begin{array}{c}\text { Radiative and } \\
\text { pair producing variants }\end{array}$ \\
\hline \hline Coulomb scattering & Bremsstrahlung \\
$p_{1} p_{2} \longrightarrow p_{1}^{\prime} p_{2}^{\prime}$ & $p_{1} p_{2} \leftrightarrow p_{1}^{\prime} p_{2}^{\prime} \gamma$ \\
$p e^{ \pm} \longrightarrow p^{\prime} e^{ \pm \prime}$ & $p e^{ \pm} \leftrightarrow p^{\prime} e^{ \pm \prime} \gamma$ \\
\hline & $p e_{1}^{ \pm} \leftrightarrow p^{\prime} e_{1}^{ \pm \prime} e^{ \pm} e^{\mp}$ \\
\hline Single Compton & Double Compton \\
$p \gamma \longrightarrow p^{\prime} \gamma^{\prime}$ & $p \gamma \leftrightarrow p^{\prime} \gamma^{\prime} \gamma^{\prime \prime}$ \\
\hline & $p \gamma \leftrightarrow p^{\prime} e^{ \pm} e^{\mp}$ \\
\hline \hline
\end{tabular}

TABLE II: Microphysical processes in the pair plasma involving protons.

In the range of energies (11) the radiative energy density can be approximated as $\rho_{r} \sim$ $n_{-} m c^{2}$, and then we have for concentrations $n_{p} \sim n_{-} \mathbf{B} \frac{m}{M}$. If protons and electrons are at the same temperature then from the equality of the kinetic energy of a proton $\epsilon_{k, p}=\frac{M v_{p}^{2}}{2}$ and the one of an electron $\epsilon_{k,-} \sim m c^{2}$ we have $\frac{v_{p}}{c} \sim \sqrt{\frac{m}{M}}$, therefore protons are indeed nonrelativistic.

In presence of protons additional binary reactions consist of Coulomb collisions between electrons (positrons) and protons, scattering of protons on protons and Compton scattering of protons. Additional triple reactions are radiative variants of these reactions, see Table II and Appendix D.

Protons can be thermalized in two ways: either in a two-step process first between themselves and then by electron/positron-proton collisions, or just by the latter mechanism. The rate of proton-proton collisions is a factor $\sqrt{\frac{m}{M}} \frac{n_{p}}{n_{-}} \sim \mathbf{B}\left(\frac{m}{M}\right)^{3 / 2}$ smaller than the rate of electron-electron collisions, see (D15). The rate of proton-electron/positron collisions is a factor $\frac{\epsilon}{M c^{2}} \sim \frac{m}{M}$ smaller than the one of electron-electron collisions, see (D11). Therefore, for B $>\sqrt{\frac{m}{M}}$ proton-proton collisions are faster, while for $\mathbf{B}<\sqrt{\frac{m}{M}}$ proton-electron/positron ones predominate. 


\section{THE DISCRETIZATION PROCEDURE AND THE COMPUTATIONAL SCHEME}

In order to solve equations (4) we use a finite difference method by introducing a computational grid in the phase space to represent the distribution functions and to compute collisional integrals following [39]. Our goal is to construct the scheme implementing energy, baryon number and electric charge conservation laws, see Appendix A, For this reason we prefer to use in the code, instead of distribution functions $f_{i}$, the spectral energy densities

$$
E_{i}\left(\epsilon_{i}\right)=\frac{4 \pi \epsilon_{i}^{3} \beta_{i} f_{i}}{c^{3}}
$$

where $\beta_{i}=\sqrt{1-\left(m_{i} c^{2} / \epsilon_{i}\right)^{2}}$, in the phase space $\epsilon_{i}$. Then

$$
\epsilon_{i} f_{i}(\mathbf{p}, t) d \mathbf{r} d \mathbf{p}=\frac{4 \pi \epsilon^{3} \beta_{i} f_{i}}{c^{3}} d \mathbf{r} d \epsilon_{i}=E_{i} d \mathbf{r} d \epsilon_{i}
$$

is the energy in the volume of the phase space $d \mathbf{r} d \mathbf{p}$. The number density of particles of type " $i$ " is given by

$$
n_{i}=\int f_{i} d \mathbf{p}=\int \frac{E_{i}}{\epsilon_{i}} d \epsilon_{i}, \quad d n_{i}=f_{i} d \mathbf{p},
$$

while the corresponding energy density is

$$
\rho_{i}=\int \epsilon_{i} f_{i} d \mathbf{p}=\int E_{i} d \epsilon_{i}
$$

We can rewrite Boltzmann equations (41) in the form

$$
\frac{1}{c} \frac{\partial E_{i}}{\partial t}=\sum_{q}\left(\tilde{\eta}_{i}^{q}-\chi_{i}^{q} E_{i}\right),
$$

where $\tilde{\eta}_{i}^{q}=\left(4 \pi \epsilon_{i}^{3} \beta_{i} / c^{3}\right) \eta_{i}^{q}$.

We introduced the computational grid for phase space $\left\{\epsilon_{i}, \mu, \phi\right\}$, where $\mu=\cos \vartheta$, $\vartheta$ and $\phi$ are angles between radius vector $\mathbf{r}$ and the particle momentum $\mathbf{p}$. The zone boundaries

are $\epsilon_{i, \omega \mp 1 / 2}, \mu_{k \mp 1 / 2}, \phi_{l \mp 1 / 2}$ for $1 \leq \omega \leq \omega_{\max }, 1 \leq k \leq k_{\max }, 1 \leq l \leq l_{\max }$. The length of the $i$-th interval is $\Delta \epsilon_{i, \omega} \equiv \epsilon_{i, \omega+1 / 2}-\epsilon_{i, \omega-1 / 2}$. On the finite grid the functions (19) become

$$
E_{i, \omega} \equiv \frac{1}{\Delta \epsilon_{i, \omega}} \int_{\Delta \epsilon_{i, \omega}} d \epsilon E_{i}(\epsilon)
$$

Now we can replace the collisional integrals in (22) by the corresponding sums.

After this procedure we get the set of ordinary differential equations (ODE's), instead of the system of partial differential equations for the quantities $E_{i, \omega}$ to be solved. There 
are several characteristic times for different processes in the problem, and therefore our system of differential equations is stiff. Under these conditions eigenvalues of Jacobi matrix differs significantly, and the real parts of eigenvalues are negative. We use Gear's method [47] to integrate ODE's numerically. This high-order implicit method was developed for the solution of stiff ODE's.

In our method exact energy conservation law is satisfied. For binary interactions the particles number conservation law is satisfied as we adopt interpolation of grid functions $E_{i, \omega}$ inside the energy intervals.

\section{NUMERICAL RESULTS}

In what follows we consider in details three specific cases. In the first two cases our grid consists of 60 energy intervals and $16 \times 32$ intervals for two angles $\vartheta$ and $\phi$ characterizing the direction of the particle momentum. In the third case we have 40 energy intervals.

\section{A. Case I}

We take the following initial conditions: flat initial spectral densities $E_{i}\left(\epsilon_{i}\right)=$ const, total energy density $\rho=10^{24} \mathrm{erg} / \mathrm{cm}^{3}$. Plasma is dominated by photons with small amount of electron-positron pairs, the ratio between energy densities in photons and in electronpositron pairs $\zeta=\rho_{ \pm} / \rho_{\gamma}=10^{-5}$. Baryonic loading parameter $\mathbf{B}=10^{-3}$, corresponding to $\rho_{p}=2.7 \times 10^{18} \mathrm{erg} / \mathrm{cm}^{3}$. The energy density in each component of plasma changes, as can be seen from fig. 1, keeping constant the total energy density shown by dotted line in fig. 1, as the energy conservation requires. As early as at $10^{-23}$ sec the energy starts to redistribute between electrons and positrons from the one hand and photons from the other hand essentially by the pair-creation process. This leads to equipartition of energies between these particles at $3 \times 10^{-15} \mathrm{sec}$. Concentrations of pairs and photons equalize at $10^{-14} \mathrm{sec}$, as can be seen from fig. 2. From this moment temperatures and chemical potentials of electrons, positrons and photons tend to be equal, see fig. 3 and fig. 4 respectively, and it corresponds to the approach to kinetic equilibrium.

This is quasi-equilibrium state since total number of particles is still approximately conserved, as can be seen from fig. 2, and triple interactions are not yet efficient. At the 


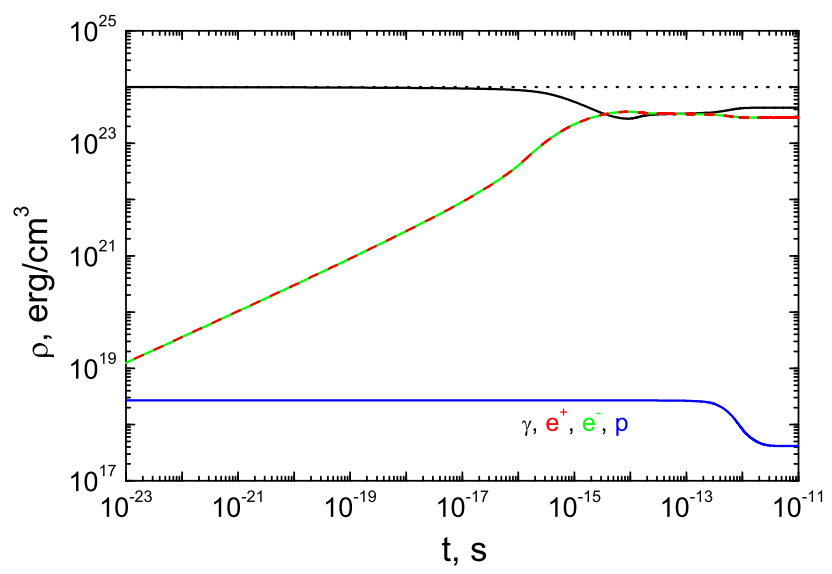

FIG. 1: Depencence on time of energy densities of electrons (green), positrons (red), photons (black) and protons (blue) for initial conditions I. Total energy density is shown by dotted black line. Interaction between pairs and photons operates on very short timescales up to $10^{-23}$ sec. Quasi-equilibrium state is established at $t_{\mathrm{k}} \simeq 10^{-14} \mathrm{sec}$ which corresponds to kinetic equilibrium for pairs and photons. Protons start to interact with then as late as at $t_{\mathrm{th}} \simeq 10^{-13} \mathrm{sec}$.

moment $t_{1}=4 \times 10^{-14} \mathrm{sec}$, shown by the vertical line on the left in fig. 3 and fig. 4, the temperature of photons and pairs is $\theta_{\mathrm{k}} \simeq 1.5$, while the chemical potentials of these particles are $\nu_{\mathrm{k}} \simeq-7$. Concentration of protons is so small that their energy density is not affected by the presence of other components; also proton-proton collisions are inefficient. In other words, protons do not interact yet and their spectra are not yet of equilibrium form, see fig. 5. The temperature of protons start to change only at $10^{-13} \mathrm{sec}$, when proton-electron Coulomb scattering becomes efficient.

As can be seen from fig. 4, the chemical potentials of electrons, positrons and photons evolved by that time due to triple interactions. Since chemical potentials of electrons, positrons and photons were negative, the particles were in deficit with respect to the thermal state. This caused the total number of these particles to increase and consequently the temperature to decrease. The chemical potential of photons reaches zero at $t_{2}=10^{-12}$ sec, shown by the vertical line on the right in fig. 3 and fig. 4, which means that electrons, positrons and photons are now in thermal equilibrium. However, protons are not yet in equilibrium with other particle since their spectra are not thermal, as shown in the lower 


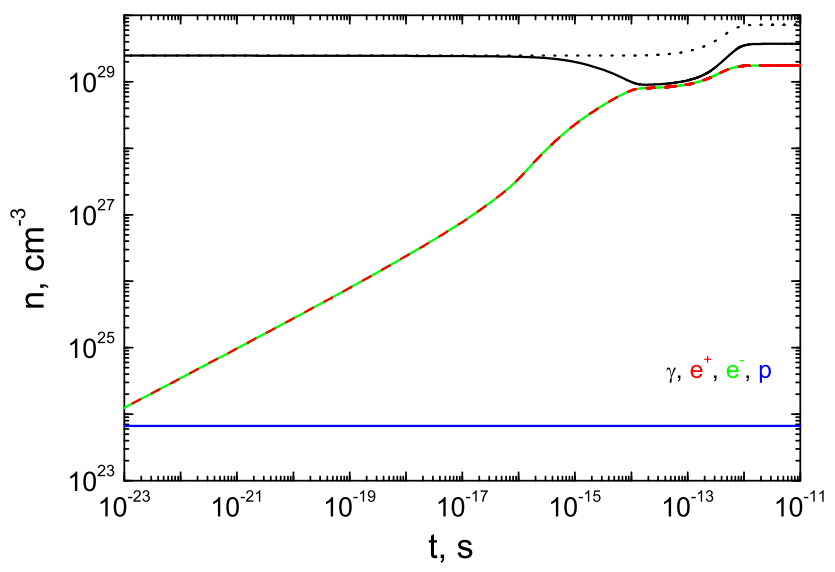

FIG. 2: Depencence on time of concentrations of electrons (green), positrons (red), photons (black) and protons (blue) for initial conditions I. Total number density is shown by dotted black line. In this case kinetic equilibrium between electrons, positrons and photons is reached at $t_{\mathrm{k}} \simeq 10^{-14}$ sec. Protons join thermal equilibrium with other particles at $t_{\text {th }} \simeq 4 \times 10^{-12}$ sec.

part of fig. 5 .

Finally, the proton component thermalize with other particles at $4 \times 10^{-12} \mathrm{sec}$, and from that moment plasma is characterized by unique temperature, $\theta_{\mathrm{th}} \simeq 0.48$ as fig. 3 clearly shows. Protons have final chemical potential $\nu_{p} \simeq-12.8$.

This state is characterized by thermal distribution of all particles as can be seen from fig. 6. There initial flat as well as final spectral densities are shown together with fits of particles spectra with the values of the common temperature and the corresponding chemical potentials in thermal equilibrium.

\section{B. Case II}

We take the following initial conditions: power law spectral densities $E_{i}\left(\epsilon_{i}\right)$ for protons, electrons and positrons with initial energy densities $\rho_{p}=2.8 \times 10^{22} \mathrm{erg} / \mathrm{cm}^{3}, \rho_{-}=1.5 \times 10^{24}$ $\mathrm{erg} / \mathrm{cm}^{3}, \rho_{+}=1.5 \times 10^{21} \mathrm{erg} / \mathrm{cm}^{3}$, respectively. We chosen flat spectral density for photons with $\rho_{\gamma}=2.8 \times 10^{24} \mathrm{erg} / \mathrm{cm}^{3}$. Initial baryonic loading parameter is set to $\mathbf{B}=608$, corresponding to a matter-dominated plasma, unlike the previous case. As in the case I, the most rapid reaction is electron-positron pair creation which starts to change the energy 


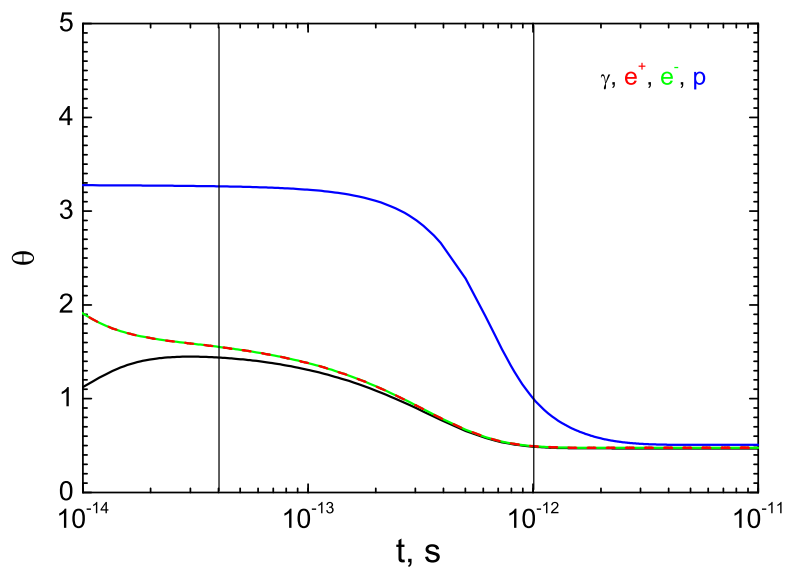

FIG. 3: Depencence on time of dimensionless temperature of electrons (green), positrons (red), photons (black) and protons (blue) for initial conditions I. The temperature for pairs and photons acquires physical meaning only in kinetic equilibrium at $t_{\mathrm{k}} \simeq 10^{-14} \mathrm{sec}$. Protons are cooled by the pair-photon plasma and acquire common temperature with it as late as at $t_{\text {th }} \simeq 4 \times 10^{-12}$ sec.

density of positrons at $10^{-20} \mathrm{sec}$, see fig. 7. Initially most energy is in photons, followed by electrons and protons. In the course of the evolution the energy gets redistributed in such a way that in the final state most energy is transferred first to the electrons, then follow the protons, the photons and finally the positrons. In fig. 8 one can see that number densities of electrons and protons are almost equal with chosen heavy proton loading. Concentrations of particles almost do not change during evolution towards thermal equilibrium.

Temperatures and chemical potentials of particles are shown in fig. 9] and 10 respectively. Kinetic equilibrium is established at around $8 \times 10^{-15} \mathrm{sec}$, marked by the vertical line. The temperature of pairs and photons at that moment is $\theta_{\mathrm{k}} \simeq 0.53$, while the chemical potentials of these particles are $\nu_{-} \simeq 1, \nu_{+} \simeq-0.9, \nu_{\gamma} \simeq 0.1$. Notice that chemical potentials of electrons and positrons are almost equal in magnitude and opposite in kinetic equilibrium, see fig. 10. At this moment protons are not yet in equilibrium with the rest of plasma but already established kinetic equilibrium with themselves with the temperature $\theta_{p} \simeq 0.18$ and the chemical potential $\nu_{p} \simeq-2$. The common temperature is reached at the moment $10^{-13}$ sec, which corresponds to thermal equilibrium. Final values of temperature is $\theta_{\text {th }} \simeq 0.47$, while chemical potentials are $\nu_{ \pm} \simeq \mp 1, \nu_{p} \simeq-4.7$. 


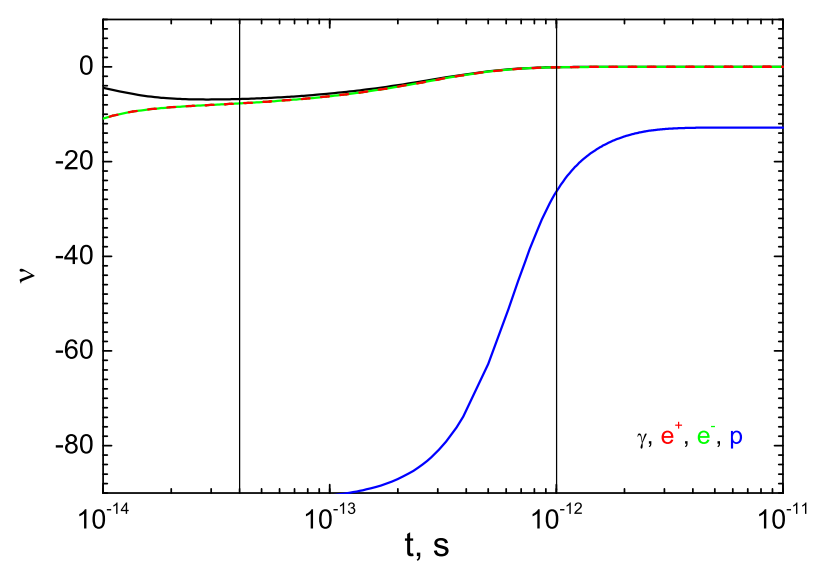

FIG. 4: Depencence on time of dimensionless chemical potential of electrons (green), positrons (red), photons (black) and protons (blue) for initial conditions I. The chemical potential for pairs and photons acquires physical meaning only in kinetic equilibrium at $t_{\mathrm{k}} \simeq 10^{-14}$ sec, while for protons this happens at $t_{\mathrm{th}} \simeq 4 \times 10^{-12}$ sec. At this time chemical potential of photons has evolved to zero and thermal equilibrium has been already reached.

The share of the proton energy density in the total energy density increased in course of time, see fig. 7, causing an increase in the baryonic loading parameter which reached in thermal equilibrium the value $\mathbf{B}=780$.

Since concentration of protons is chosen to be large, proton-proton collisions become more important than proton-electron/positron collisions, in contrast to the case I. In fact, protons reached equilibrium temperature already at $10^{-16} \mathrm{sec}$, while they start to interact with electrons and positrons only at $10^{-15}$ sec.

\section{Case III}

We take the following initial conditions: the initial ratio between concentrations of electrons and protons is $\varsigma=n_{p} / n_{-}=10^{-3}$. The total energy density is chosen in such a way that the final temperature in thermal equilibrium be $\theta_{\mathrm{th}}=2$. We set up flat initial spectrum for photons $E_{\gamma}\left(\epsilon_{i}\right)=$ const, and power law spectra for the pairs $E_{ \pm}\left(\epsilon_{ \pm}\right) \propto\left[\epsilon_{ \pm}-m c^{2}\right]^{-2}$ and protons $E_{p}\left(\epsilon_{p}\right) \propto\left[\epsilon_{p}-M c^{2}\right]^{-4}$. Finally, the ratio of initial and final concentrations of positrons is chosen to be $n_{+}=10^{-1} n_{+}^{\text {th }}$. Given these initial conditions the baryon loading 


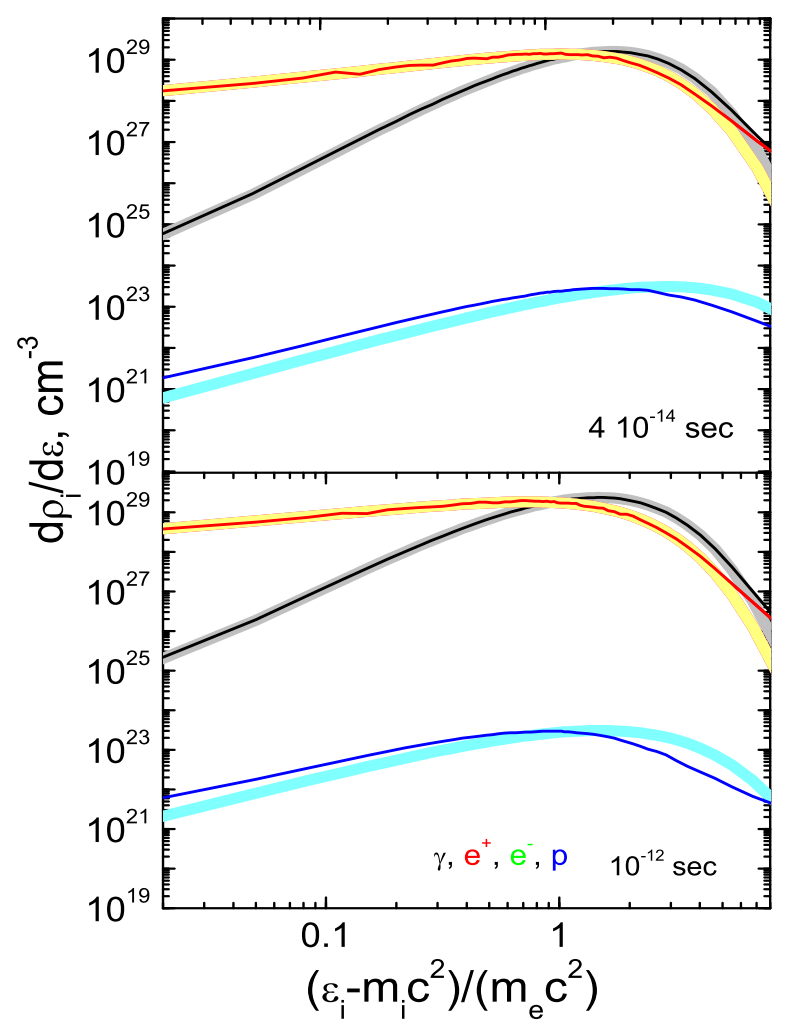

FIG. 5: Spectral density as function of particle energy for electrons (green), positrons (red), photons (black) and protons (blue) for initial conditions I at intermediate time moments $t_{1}=4 \times 10^{-14}$ sec (upper figure) and $t_{2}=10^{-12} \mathrm{sec}$ (lower figure). Fits of the spectra with chemical potentials and temperatures corresponding to thermal equilibrium state are also shown by yellow (electrons and positrons), grey (photons) and light blue (protons) thick lines. The upper figure shows the spectra when kinetic equilibrium is established for the first time between electrons, positrons and photons while the lower figure shows the spectra at thermal equilibrium between these particles. On both figures protons are not yet in equilibrium neither with themselves nor with other particles.

parameter is $\mathbf{B}=0.2$. The initial conditions are chosen in a way to get larger temperature in thermal equilibrium, than in previous cases. Unlike the case II, the spectrum of protons is chosen steeper than the spectrum of pairs in order to make them colder in kinetic equilibrium.

Equipartition of energies between pairs and photons occurs earlier than in the case I, at around $10^{-17} \mathrm{sec}$, see fig. 12, since now concentrations of particles are higher. Concentrations 


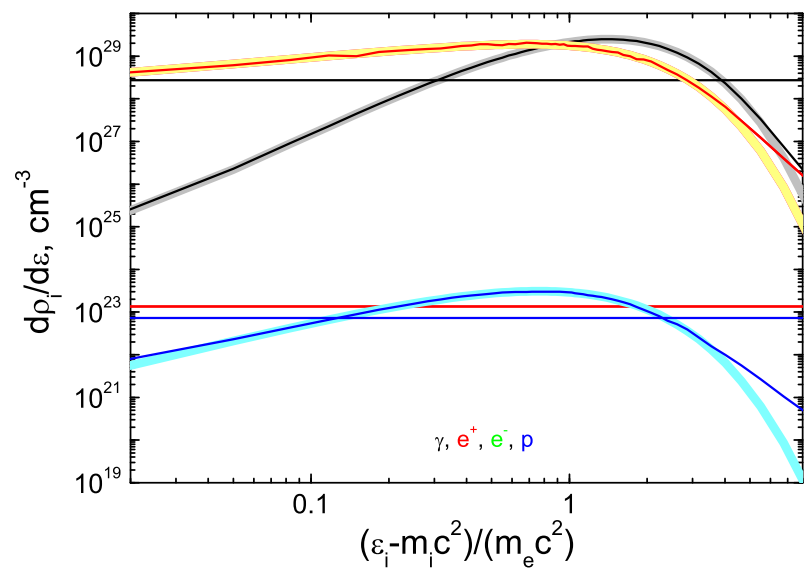

FIG. 6: Spectral density as function of particle energy are shown as before at initial and final moments of the computations. The final photon spectrum is black body one.

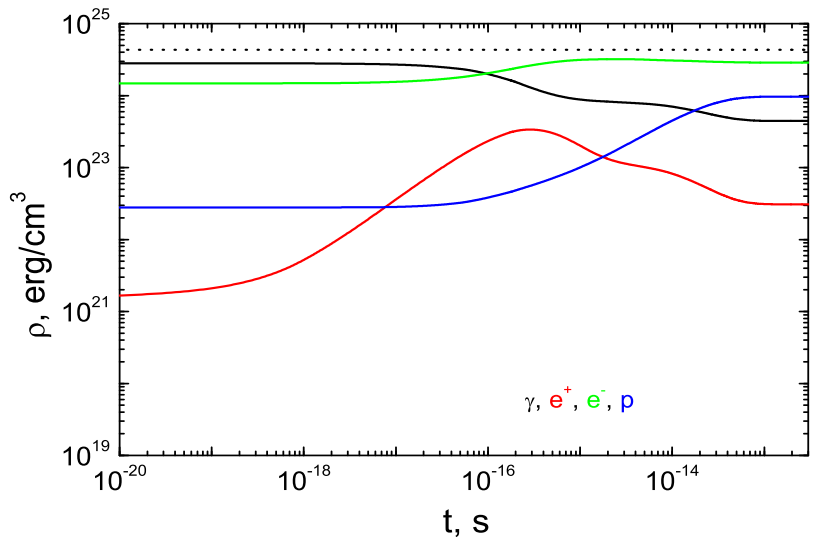

FIG. 7: Depencence on time of energy densities for initial conditions II. Colors are as in the case I. Protons start to interact with other particles as late as at $t \simeq 10^{-16} \mathrm{sec}$.

of pairs and photons equalize at $3 \times 10^{-17}$ sec, see fig. 13. As in the case I, from this moment temperatures and chemical potentials of electrons, positrons and photons tend to be equal, see fig. 14 and fig. 15 respectively, leading to kinetic equilibrium at around $t_{\mathrm{k}} \simeq 10^{-16}$ sec.

At the moment $t_{\mathrm{k}}$, shown by the vertical line on the left in fig. 14 and fig. 15, the temperature of photons and pairs is $\theta_{\mathrm{k}} \simeq 2.2$, the chemical potentials of these particles are $\nu_{\mathrm{k}} \simeq-1.1$, while the temperature of protons, having well established spectrum by this time, 


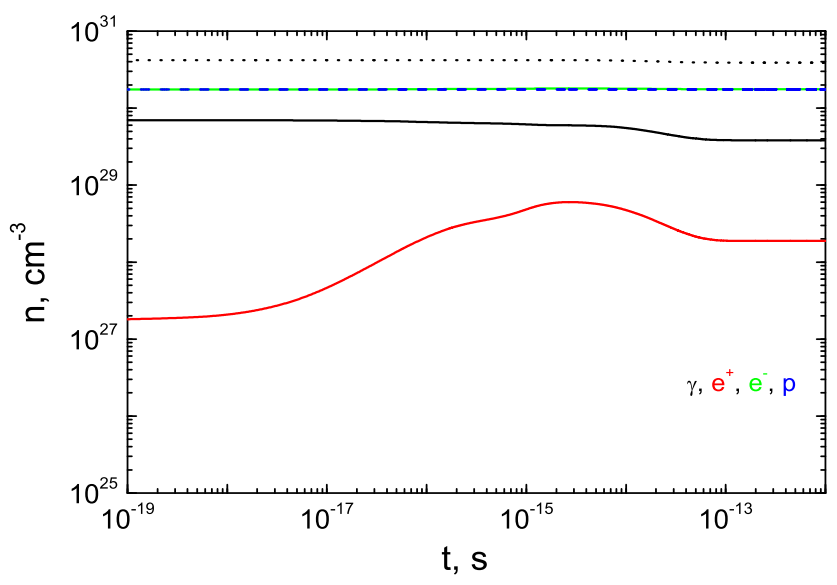

FIG. 8: Depencence on time of concentrations for initial conditions II. Colors are as in the case I.

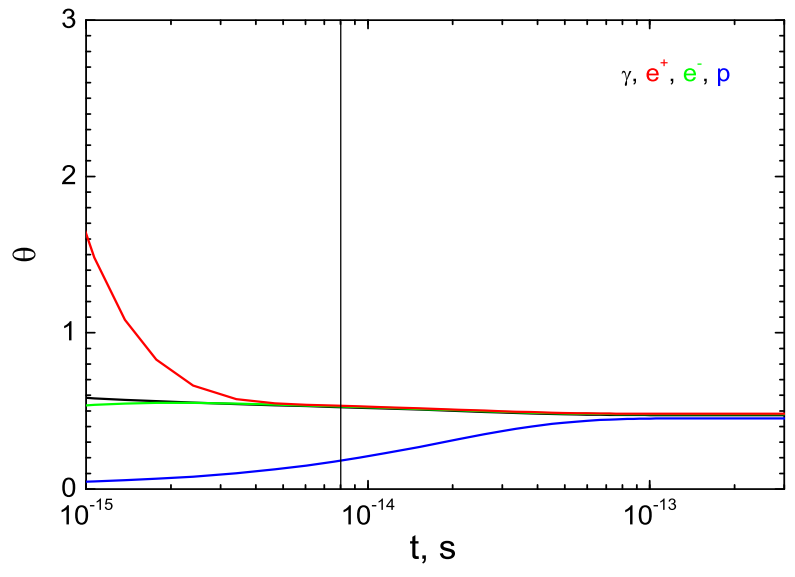

FIG. 9: Depencence on time of dimensionless temperature for initial conditions II. Colors are as in the case I. The pair-photon plasma is heating protons. Protons join thermal equilibrium at $t_{\mathrm{th}} \simeq 10^{-13} \mathrm{sec}$.

is just $\theta_{p} \simeq 0.09$.

Thermal equilibrium is reached in the electron-positron-photon plasma at around $t_{\mathrm{th}} \simeq$ $4 \times 10^{-15}$ sec, shown by the vertical line on the right of fig. 14 and fig. 15 . Only at $4 \times 10^{-14}$ sec the temperature becomes common also with protons which are heated up during this time. The temperature at this final stage is $\theta_{\mathrm{th}} \simeq 2$ while the chemical potential of protons 


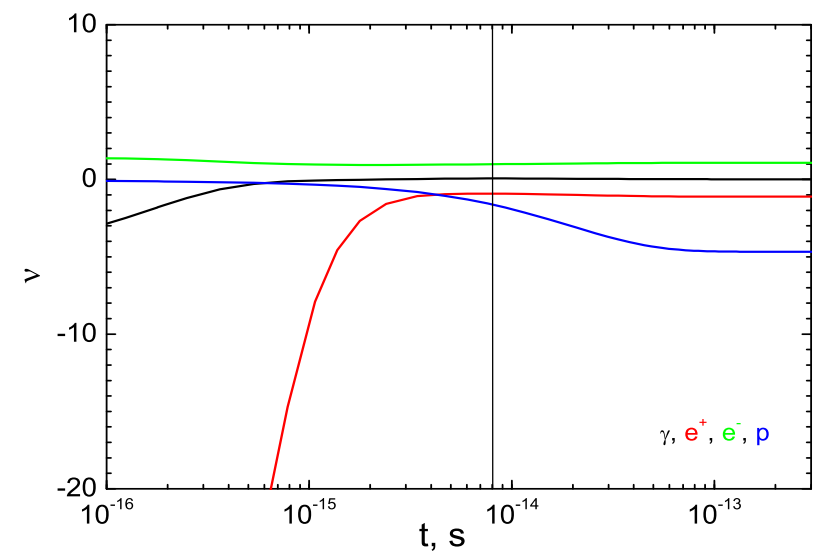

FIG. 10: Depencence on time of dimensionless chemical potential for initial conditions II. Colors are as in the case I. The chemical potential of photons is almost zero in kinetic equilibrium. The chemical potentials of electrons and positrons are almost equal and opposite in kinetic equilibrium, to maintain electric neutrality.

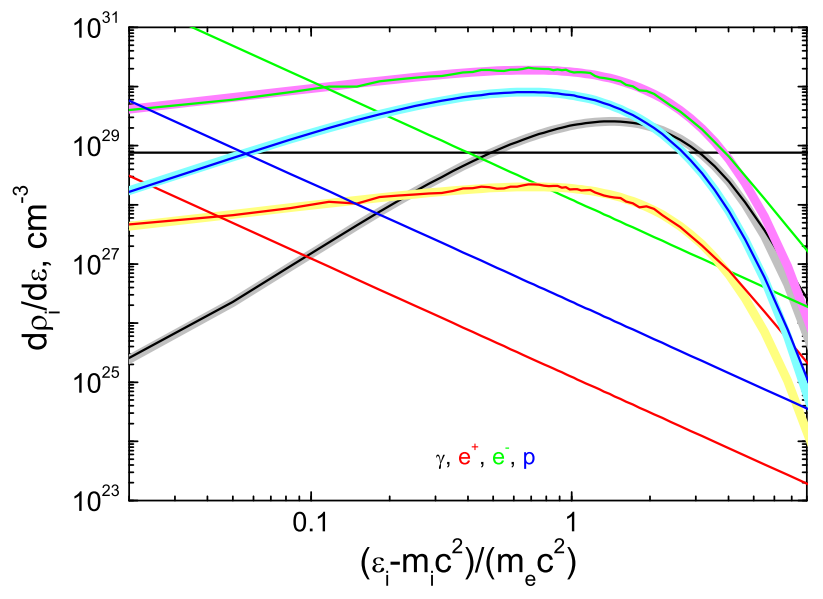

FIG. 11: Initial and final spectral density as function of particle energy for initial conditions II. Fits of the final spectra with chemical potentials and temperatures are also shown.

is $\nu_{p} \simeq-33$. Initial as well as final spectra are shown in fig. 16.

Since chemical potentials and temperatures approach their values in thermal equilibrium exponentially, i.e. $\sim \exp \left(-t / \tau_{\text {ch.eq }}\right)$, we determined the relaxation time constant $\tau_{\text {ch.eq }}$ for 


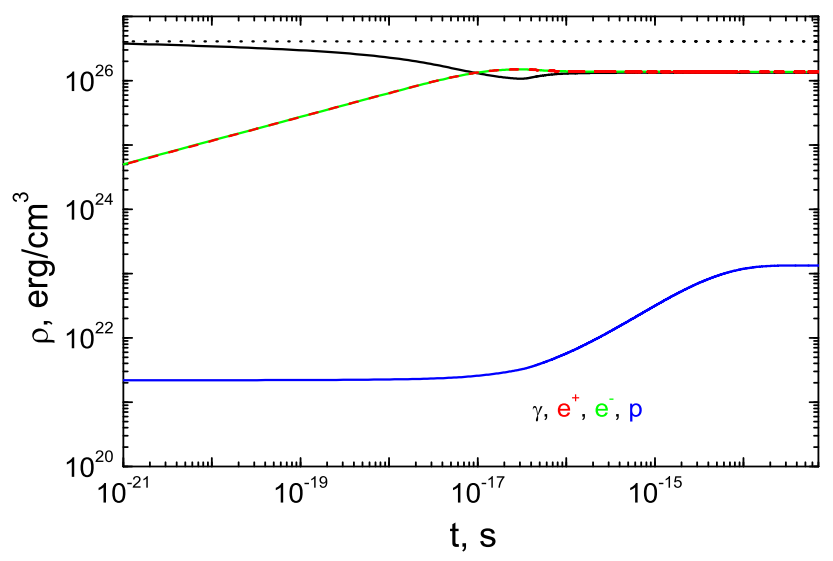

FIG. 12: Depencence on time of energy densities for initial conditions III. Colors are as in the case I. Protons start to interact with other particles at about $10^{-17}$ sec.

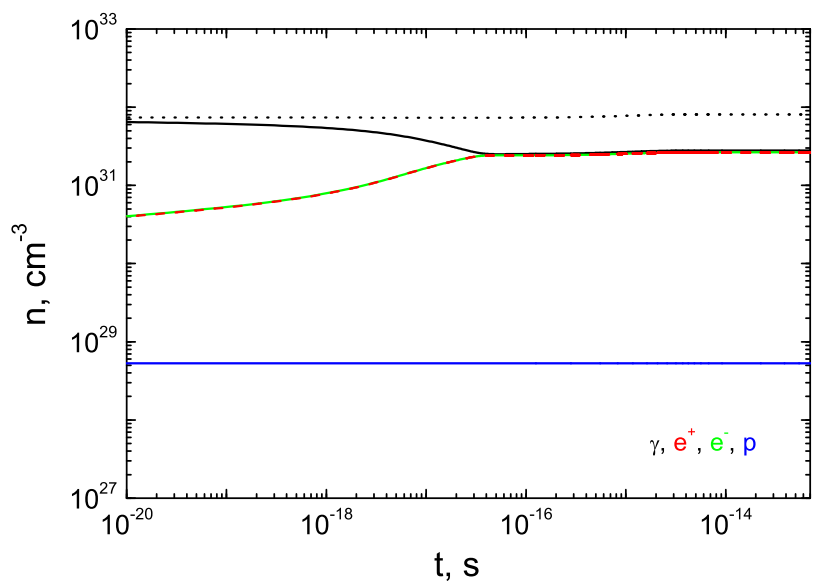

FIG. 13: Depencence on time of concentrations for initial conditions III. Colors are as in the case I.

each of the cases considered from

$$
\tau_{\text {ch.eq }}=\lim _{t \rightarrow \infty}\left[(F(t)-F(\infty))\left(\frac{d F}{d t}\right)^{-1}\right],
$$

where the fugacity for a given sort of particle is given by (13). Our results are presented in Tab. III. 


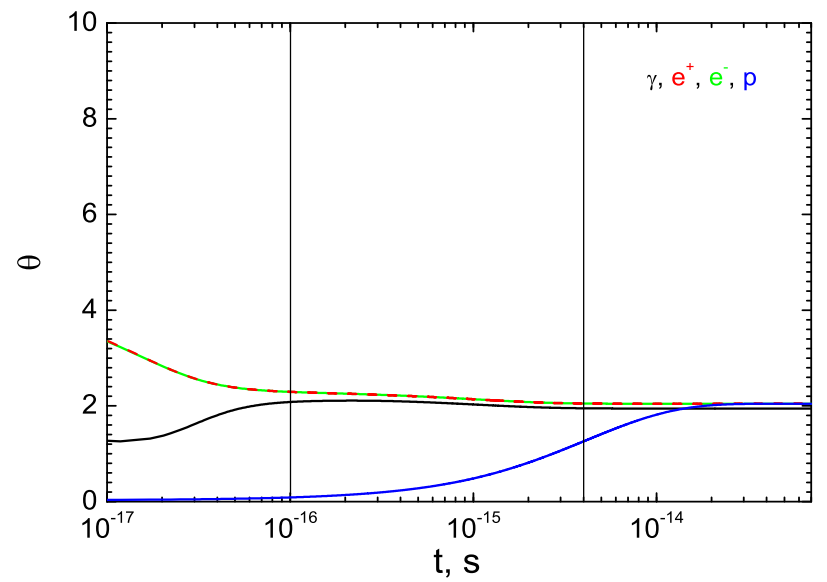

FIG. 14: Depencence on time of dimensionless temperature for initial conditions III. Colors are as in the case I. Pairs and photons acquire the temperature at $t_{\mathrm{k}} \simeq 10^{-16}$ sec.

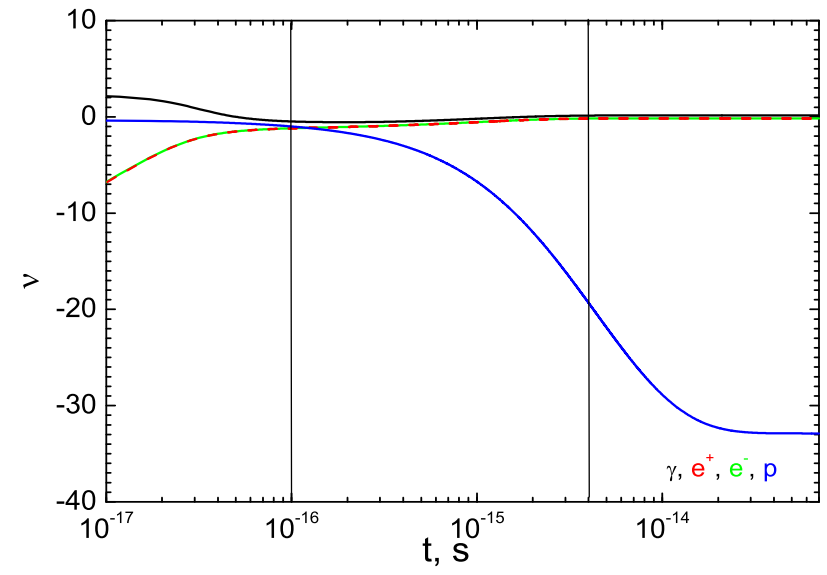

FIG. 15: Depencence on time of dimensionless chemical potential for initial conditions III. Colors are as in the case I. The chemical potentials equalize at $t_{\mathrm{k}} \simeq 10^{-16}$ sec.

\section{DISCUSSION AND CONCLUSIONS}

Results presented above clearly show the existence of two types of equilibrium: the kinetic and the thermal ones. Kinetic equilibrium in pair-photon plasma occurs when Ehlers [46] balance conditions $(\overline{\mathrm{B} 6}),(\mathrm{B} 9)$ and $(\overline{\mathrm{B} 12})$ are satisfied so that pair-creation, Compton and 


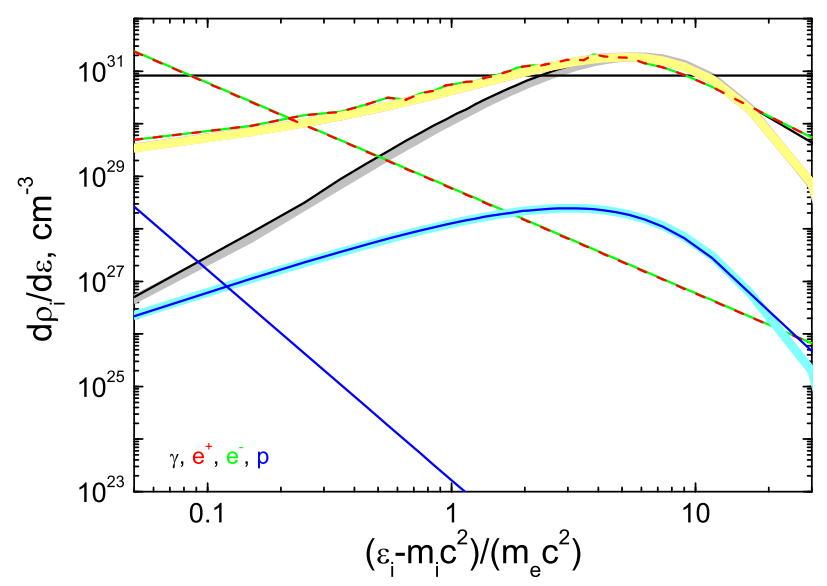

FIG. 16: Initial and final spectral density as function of particle energy for initial conditions III. The spectrum of protons is chosen to be steeper than the one of electrons and positrons. Fits of the final spectra with chemical potentials and temperatures are also shown.

\begin{tabular}{|c|c|c|c|}
\hline \hline & I & II & III \\
\hline \hline$\tau_{\text {ch.eq }}^{ \pm, \gamma}$, sec & $2.2 \times 10^{-13}$ & $1.8 \times 10^{-14}$ & $9.5 \times 10^{-16}$ \\
\hline$\tau_{\text {ch.eq }}^{p}$, sec & $6 \times 10^{-13}$ & $1.8 \times 10^{-14}$ & $5.5 \times 10^{-15}$ \\
\hline \hline
\end{tabular}

TABLE III: Relaxation time constant for cases I-III.

Bhabha/Møller scattering processes all come in detailed balance. The electron-positronphoton plasma then is described by common temperature and nonzero chemical potentials which are given by $(\underline{\mathrm{B} 23}),(\underline{\mathrm{B} 24})$ and $(\underline{\mathrm{B} 25}),(\underline{\mathrm{B} 26})$. Protons at this stage may or may not have yet established equilibrium with the spectrum (15), depending on the value of the baryon loading parameter $\mathbf{B}$. When $\mathbf{B}$ is small, as in the case I, proton-proton collision are inefficient since the rate (D15) is much smaller than (D11), and the proton spectrum is shaped up by the proton-electron/positron collisions, reaching equilibrium form at a timescale given by (D11), when other particles are already in thermal equilibrium. When $\mathbf{B}$ is large, as in the case II, protons have established their equilibrium temperature at a timescale given by (D15), prior to the moment when kinetic equilibrium in the pair-photon plasma is established.

As we have seen, the final spectra are completely insensitive to the initial spectra, chosen to be flat as in the case I, power-law as in the case II, or thermal ones. 
The meaning of non-zero chemical potentials in kinetic equilibrium can be understood as follows. The existence of a non-null chemical potential for photons indicates the departure of the distribution function from the one corresponding to the thermal equilibrium. Negative value of the chemical potential generates an increase of the number of particles in order to approach the one corresponding to the thermal equilibrium state. Positive value of the chemical potential leads to the opposite effect, decreasing the number of particles. Then, since the total number of particles increases (or decreases), the energy is shared between larger (or smaller) number of particles and the temperature decreases (or increases). Clearly, as thermal equilibrium is approached, the chemical potential of photons tends to zero, while the chemical potentials of electrons and positrons are given by (B27), to guarantee an overall charge neutrality.

One of the basic assumptions in this work is that triple interactions are slower than binary ones, allowing to use reaction rates for triple interactions in kinetic equilibrium, explicitly depending solely on temperature, chemical potentials and concentrations of particles. For pure electron-positron plasma in the range of energies of interest (11) there is a hierarchy of relevant timescales: binary interactions are clearly faster than triple ones. However, when protons are also present, the proton-proton timescale may be shorter or longer than the corresponding binary interactions timescales for the pure pair plasma. This violates our assumption and therefore leads to loss of quantitative accuracy, although still keeping qualitative results valid. In order to overcome this difficulty and produce quantitatively precise results exact QED matrix elements must be used for calculation of emission and absorption coefficients.

Notice that there is some discrepancy between our final spectra and their thermal fits for high energy. This is due to poor energy resolution with adopted grid. The result converges with higher resolutions, but it is limited by the available computer memory. In addition, the code is quite time-consuming and processor time increases with number of operation as third power of the number of energy intervals.

In order to resolve proton-electron/positron scattering the number of energy intervals should be increased as $M / m$ comparing to the case of pure pair plasma. Even using inhomogeneous energy grid with uniform energy step up to the peak of the spectrum $d \rho / d \varepsilon$ and decreasing energy step as $\varepsilon^{-1}$ for higher energies, we have obtained acceptable results with about $10^{3}$ intervals for this reaction. Using such fine grid is impossible in practice. 
On the other hand, a small parameter $m / M$ expansion can be adopted. In this way we have introduced the mass scaling, described in Appendix G, which gives quite good accuracy for about $10^{2}$ intervals in energy with inhomogeneous grid, described above. Finally, it is important to stress that our code allows solution of the Boltzmann equations for long time intervals and timescales, which may differ up to 10 orders of magnitude, from electronpositron creation and annihilation process up to proton-electron/positron scattering, see fig. 2, unlike approaches based on Monte-Carlo technique [45]. This gives us the possibility to follow thermalization process up to reaching steady solution, i.e. thermal equilibrium.

The assumption of the constancy of the energy density is only valid if the following three conditions are satisfied:

- plasma is optically thick for photons. This leads to the constraint on the spatial dimensions $R_{0} \gg\left(n_{\mathrm{th}} \sigma_{T}\right)^{-1} \sim 10^{-5} \mathrm{~cm}$.

- neutrinos are not produced. This gives the constraint on the temperature $\theta \ll 7 \times 10^{2}$.

- plasma does not expand. Given $t_{\mathrm{dyn}}=\left(\frac{1}{R} \frac{d R}{d t}\right)^{-1} \gg t_{\mathrm{th}}$, this leads to $R_{0} \gg 10^{-2} \mathrm{~cm}$.

To summarize, we have considered the evolution of initially nonequilibrium optically thick electron-positron-photon plasma with proton loading up to reaching thermal equilibrium on a timescale $t_{\mathrm{th}} \lesssim 10^{-11}$ sec. Starting from arbitrary initial conditions we obtain kinetic equilibrium, on a timescale $t_{\mathrm{k}} \lesssim 10^{-14} \mathrm{sec}$, from first principles, solving numerically the relativistic Boltzmann equation with collisional integrals computed from exact QED matrix elements.

The general theoretical framework here presented can be further applied by considering thermalization of different relativistic particles predicted by extensions of the standard model of particle physics with the lepton plasma in the early Universe. The occurence of thermalization process of electron-positron plasma in GRBs on a much shorter timescale than the characteristic acceleration time [48] is crucial. Such acceleration timescales are indeed sharply bounded (shorter than $10^{3} \mathrm{sec}$ in the laboratory frame). Determination of thermalization timescales as functions of the relevant parameters is important for high energy plasma physics [49], [50]. Finally, these results can in principle be tested in laboratory experiments aiming the generation of electron-positron pairs. 
We thank the anonymous referee for comments which improved the presentation of our results.

\section{APPENDIX A: CONSERVATION LAWS}

Conservation laws consist of baryon number, charge and energy conservations. In addition, in binary reactions particle number is conserved.

Energy conservation law can be rewritten for the spectral density

$$
\frac{d}{d t} \sum_{i} \rho_{i}=0, \quad \text { or } \quad \frac{d}{d t} \sum_{i, \omega} Y_{i, \omega}=0,
$$

where

$$
Y_{i, \omega}=\int_{\epsilon_{i, \omega}-\Delta \epsilon_{i, \omega} / 2}^{\epsilon_{i, \omega}+\Delta \epsilon_{i, \omega} / 2} E_{i} d \epsilon .
$$

Particle's conservation law in binary reactions gives

$$
\frac{d}{d t} \sum_{i} n_{i}=0, \quad \text { or } \quad \frac{d}{d t} \sum_{i, \omega} \frac{Y_{i, \omega}}{\epsilon_{i, \omega}}=0 .
$$

Since baryonic number is conserved, therefore the number density of protons is a constant

$$
\frac{d n_{p}}{d t}=0
$$

For electrically neutral plasma considered in this paper charge conservation implies (17).

APPENDIX B: DETERMINATION OF TEMPERATURE AND CHEMICAL POTENTIALS IN KINETIC EQUILIBRIUM

Consider distribution functions for photons and pairs in the most general form (15). If one supposes that reaction rate for the Bhabha scattering vanishes, i.e. there is equilibrium with respect to reaction

$$
e^{+}+e^{-} \leftrightarrow+e^{+\prime}+e^{-\prime},
$$

then the corresponding condition can be written in the following way

$$
f_{+}\left(1-f_{+}{ }^{\prime}\right) f_{-}\left(1-f_{-}{ }^{\prime}\right)=f_{+}{ }^{\prime}\left(1-f_{+}\right) f_{-}{ }^{\prime}\left(1+f_{-}\right),
$$


where Bose-Einstein enhancement along with Pauli blocking factors are taken into account for generality, it can be shown that electrons and positrons have the same temperature

$$
\theta_{+}=\theta_{-} \equiv \theta_{ \pm}
$$

and they have arbitrary chemical potentials.

With (B7) analogous consideration for the Compton scattering

$$
e^{ \pm}+\gamma \leftrightarrow+e^{ \pm \prime}+\gamma^{\prime}
$$

gives

$$
f_{ \pm}\left(1-f_{ \pm}{ }^{\prime}\right) f_{\gamma}\left(1+f_{\gamma}{ }^{\prime}\right)=f_{ \pm}{ }^{\prime}\left(1-f_{ \pm}\right) f_{\gamma}{ }^{\prime}\left(1+f_{\gamma}\right),
$$

and leads to equality of temperatures of pairs and photons

$$
\theta_{ \pm}=\theta_{\gamma} \equiv \theta_{k},
$$

with arbitrary chemical potentials. If, in addition, reaction rate in the pair-creation and annihilation process

$$
e^{ \pm}+e^{\mp} \leftrightarrow \gamma+\gamma^{\prime}
$$

vanishes too, i.e. there is equilibrium with respect to pair production and annihilation, with the corresponding condition,

$$
f_{+} f_{-}\left(1+f_{\gamma}\right)\left(1+f_{\gamma}{ }^{\prime}\right)=f_{\gamma} f_{\gamma}{ }^{\prime}\left(1-f_{+}\right)\left(1-f_{-}\right),
$$

it turns out that also chemical potentials of pairs and photons satisfy the following condition

$$
\nu_{+}+\nu_{-}=2 \nu_{\gamma}
$$

However, since, generally speaking, $\nu_{\gamma} \neq 0$ the condition (B13) does not imply $\nu_{+}=\nu_{-}$. These considerations were for the first time applied by Ehlers in [46], see also [51], and we will call (B6),(B9) and (B12) the Ehlers balance conditions.

Analogous consideration for the detailed balance conditions in different reactions lead to relations between temperatures and chemical potentials summarized in table IV.

The timescales of pair production and annihilation processes as well as Compton scattering are nearly equal in the range of energies of interest and are given by (14). Therefore, kinetic equilibrium is first established simultaneously for electrons, positrons and photons. 


\begin{tabular}{|c|c|c|}
\hline \hline & Interaction & Parameters of DFs \\
\hline \hline I & $e^{+} e^{-}$scattering & $\theta_{+}=\theta_{-}, \forall \nu_{+}, \nu_{-}$ \\
\hline II & $e^{ \pm} p$ scattering & $\theta_{p}=\theta_{ \pm}, \forall \nu_{ \pm}, \nu_{p}$ \\
\hline III & $e^{ \pm} \gamma$ scattering & $\theta_{\gamma}=\theta_{ \pm}, \forall \nu_{\gamma}, \nu_{ \pm}$ \\
\hline IV & pair production & $\nu_{+}+\nu_{-}=2 \nu_{\gamma}$, if $\theta_{\gamma}=\theta_{ \pm}$ \\
\hline V & Tripe interactions & $\nu_{\gamma}, \nu_{ \pm}=0$, if $\theta_{\gamma}=\theta_{ \pm}$ \\
\hline \hline
\end{tabular}

TABLE IV: Relations between parameters of equilibrium DFs fulfilling detailed balance conditions for the reactions shown in Tab. I]

They reach the same temperature, but with chemical potentials different from zero. Later on, the temperatures of this electron-positron-photon plasma and the one of protons reach a common value.

In order to find temperatures and chemical potentials we have to implement the following constraints: energy conservation (A1), particle number conservation (A3), charge conservation (17), condition for the chemical potentials (B13).

Given (15) we have for photons

$$
\frac{\rho_{\gamma}}{n_{\gamma} m c^{2}}=3 \theta_{\gamma}, \quad n_{\gamma}=\frac{1}{V_{0}} \exp \left(\frac{\nu_{\gamma}}{\theta_{\gamma}}\right) 2 \theta_{\gamma}^{3},
$$

for pairs

$$
\frac{\rho_{ \pm}}{n_{ \pm} m c^{2}}=j_{2}\left(\theta_{ \pm}\right), \quad n_{ \pm}=\frac{1}{V_{0}} \exp \left(\frac{\nu_{ \pm}}{\theta_{ \pm}}\right) j_{1}\left(\theta_{ \pm}\right)
$$

and for protons

$$
\begin{gathered}
\frac{\rho_{p}}{M n_{p} c^{2}}=1+\frac{3}{2} \frac{m}{M} \theta_{p} \\
n_{p}=\frac{1}{V_{0}} \sqrt{\frac{\pi}{2}}\left(\frac{M}{m}\right)^{3 / 2} \exp \left(\frac{\nu_{p}-\frac{M}{m}}{\theta_{p}}\right) \theta_{p}^{\frac{3}{2}}
\end{gathered}
$$

where we assumed that protons are nonrelativistic, and we denoted the Compton volume

$$
V_{0}=\frac{1}{8 \pi}\left(\frac{2 \pi \hbar}{m c}\right)^{3}
$$


and functions $j_{1}$ and $j_{2}$ are defined as

$$
\begin{aligned}
& j_{1}(\theta)=\theta K_{2}\left(\theta^{-1}\right) \rightarrow\left\{\begin{array}{cc}
\sqrt{\frac{\pi}{2}} e^{-\frac{1}{\theta} \theta^{3 / 2},} & \theta \rightarrow 0 \\
2 \theta^{3}, & \theta \rightarrow \infty
\end{array},\right. \\
& j_{2}(\theta)=\frac{3 K_{3}\left(\theta^{-1}\right)+K_{1}\left(\theta^{-1}\right)}{4 K_{2}\left(\theta^{-1}\right)} \rightarrow\left\{\begin{array}{cc}
1+\frac{3 \theta}{2}, & \theta \rightarrow 0 \\
3 \theta, & \theta \rightarrow \infty
\end{array} .\right.
\end{aligned}
$$

For pure electron-positron-photon plasma in kinetic equilibrium, summing up energy densities in $(\overline{\mathrm{B} 14}),(\mathrm{B} 15)$ and using $(\overline{\mathrm{B} 7}),(\mathrm{B} 10)$ and $(\mathrm{B} 13)$ we obtain

$$
\sum_{e^{+}, e^{-}, \gamma} \rho_{i}=\frac{2 m c^{2}}{V_{0}} \exp \left(\frac{\nu_{\mathrm{k}}}{\theta_{\mathrm{k}}}\right)\left[3 \theta^{4}+j_{1}\left(\theta_{k}\right) j_{2}\left(\theta_{\mathrm{k}}\right)\right],
$$

and analogously for number densities we get

$$
\sum_{e^{+}, e^{-}, \gamma} n_{i}=\frac{2}{V_{0}} \exp \left(\frac{\nu_{\mathrm{k}}}{\theta_{\mathrm{k}}}\right)\left[\theta_{\mathrm{k}}^{3}+j_{1}\left(\theta_{\mathrm{k}}\right)\right] .
$$

From (B21) and (B22) two unknowns, $\nu_{k}$ and $\theta_{\mathrm{k}}$ can be found.

When protons are present, in most cases the electron-positron-photon plasma reaches kinetic equilibrium first, while protons join the plasma later. In that case, the temperature of protons $\theta_{p}$ is different from the rest of particles, so while $\theta_{+}=\theta_{-}=\theta_{\gamma}=\theta_{\mathrm{k}}, \theta_{p} \neq \theta_{\mathrm{k}}$.

Then summing up energy densities in (B14), (B15) we obtain

$$
\begin{gathered}
\sum_{e^{+}, e^{-}, \gamma} \rho_{i}=\frac{m c^{2}}{V_{0}}\left\{\left[1-\frac{n_{p} V_{0}}{j_{1}\left(\theta_{\mathrm{k}}\right)} \exp \left(-\frac{\nu_{+}}{\theta_{\mathrm{k}}}\right)\right]^{\frac{1}{2}} \times\right. \\
\left.\times 6 \theta_{\mathrm{k}}^{4} \exp \left(\frac{\nu_{+}}{\theta_{\mathrm{k}}}\right)+\left[2 j_{1}\left(\theta_{k}\right) \exp \left(\frac{\nu_{+}}{\theta_{\mathrm{k}}}\right)-n_{p} V_{0}\right] j_{2}\left(\theta_{\mathrm{k}}\right)\right\},
\end{gathered}
$$

and analogously for number densities we get

$$
\begin{gathered}
\sum_{e^{+}, e^{-}, \gamma} n_{i}=\frac{1}{V_{0}}\left\{\left[1-\frac{n_{p} V_{0}}{j_{1}\left(\theta_{\mathrm{k}}\right)} \exp \left(-\frac{\nu_{+}}{\theta_{\mathrm{k}}}\right)\right]^{\frac{1}{2}} \times\right. \\
\left.\times 6 \theta_{\mathrm{k}}^{4} \exp \left(\frac{\nu_{+}}{\theta_{\mathrm{k}}}\right)+2 j_{1}\left(\theta_{\mathrm{k}}\right) \exp \left(\frac{\nu_{+}}{\theta_{\mathrm{k}}}\right)\right\} .
\end{gathered}
$$

From (B23) and (B24) two unknowns, $\nu_{+}$and $\theta_{\mathrm{k}}$ can be found. Then the rest of the chemical potentials are obtained from

$$
\begin{gathered}
\exp \left(\frac{\nu_{-}}{\theta_{\mathrm{k}}}\right)=\exp \left(\frac{\nu_{+}}{\theta_{\mathrm{k}}}\right)+\frac{n_{p} V_{0}}{j_{1}\left(\theta_{\mathrm{k}}\right)} \\
\exp \left(\frac{\nu_{\gamma}}{\theta_{\mathrm{k}}}\right)=\exp \left(\frac{\nu_{+}}{\theta_{\mathrm{k}}}\right)\left[1+\frac{n_{p} V_{0}}{j_{1}\left(\theta_{\mathrm{k}}\right)} \exp \left(-\frac{\nu_{+}}{\theta_{\mathrm{k}}}\right)\right]^{\frac{1}{2}},
\end{gathered}
$$


The temperature and chemical potential of protons can be found separately from (B16), (B17).

In thermal equilibrium $\nu_{\gamma}$ vanishes and one has

$$
\nu_{-}=\theta_{\mathrm{k}} \operatorname{arcsinh}\left[\frac{n_{p} V_{0}}{2 j_{1}\left(\theta_{\mathrm{k}}\right)}\right], \quad \nu_{+}=-\nu_{-},
$$

which both reduce to $\nu_{-}=\nu_{+}=0$ for $n_{p}=0$. At the same time, for $n_{p}>0$ one always has $\nu_{-}>0$ and $\nu_{+}<0$ in thermal equilibrium. The chemical potential of protons in thermal equilibrium is determined from (B17) for $\theta_{\mathrm{k}}=\theta_{\mathrm{th}}$, where $\theta_{\mathrm{th}}$ is the temperature in thermal equilibrium.

\section{APPENDIX C: BINARY INTERACTIONS}

\section{Compton scattering $\gamma e^{ \pm} \rightarrow \gamma^{\prime} e^{ \pm \prime}$}

The time evolution of the distribution functions of photons and pair particles due to Compton scattering may be described by [34], [52]

$$
\begin{aligned}
& \left(\frac{\partial f_{\gamma}(\mathbf{k}, t)}{\partial t}\right)_{\gamma e^{ \pm} \rightarrow \gamma^{\prime} e^{ \pm \prime}}=\int d \mathbf{k}^{\prime} d \mathbf{p} d \mathbf{p}^{\prime} V w_{\mathbf{k}^{\prime}, \mathbf{p}^{\prime} ; \mathbf{k}, \mathbf{p}}\left[f_{\gamma}\left(\mathbf{k}^{\prime}, t\right) f_{ \pm}\left(\mathbf{p}^{\prime}, t\right)-f_{\gamma}(\mathbf{k}, t) f_{ \pm}(\mathbf{p}, t)\right] \\
& \left(\frac{\partial f_{ \pm}(\mathbf{p}, t)}{\partial t}\right)_{\gamma e^{ \pm} \rightarrow \gamma^{\prime} e^{ \pm \prime}}=\int d \mathbf{k} d \mathbf{k}^{\prime} d \mathbf{p}^{\prime} V w_{\mathbf{k}^{\prime}, \mathbf{p}^{\prime} ; \mathbf{k}, \mathbf{p}}\left[f_{\gamma}\left(\mathbf{k}^{\prime}, t\right) f_{ \pm}\left(\mathbf{p}^{\prime}, t\right)-f_{\gamma}(\mathbf{k}, t) f_{ \pm}(\mathbf{p}, t)\right]
\end{aligned}
$$

where

$$
w_{\mathbf{k}^{\prime}, \mathbf{p}^{\prime} ; \mathbf{k}, \mathbf{p}}=\frac{\hbar^{2} c^{6}}{(2 \pi)^{2} V} \delta\left(\epsilon_{\gamma}-\epsilon_{ \pm}-\epsilon_{\gamma}^{\prime}-\epsilon_{ \pm}^{\prime}\right) \delta\left(\mathbf{k}+\mathbf{p}-\mathbf{k}^{\prime}-\mathbf{p}^{\prime}\right) \frac{\left|M_{f i}\right|^{2}}{16 \epsilon_{\gamma} \epsilon_{ \pm} \epsilon_{\gamma}^{\prime} \epsilon_{ \pm}^{\prime}}
$$

is the probability of the process,

$$
\begin{aligned}
\left|M_{f i}\right|^{2} & =2^{6} \pi^{2} \alpha^{2}\left[\frac{m^{2} c^{2}}{s-m^{2} c^{2}}+\frac{m^{2} c^{2}}{u-m^{2} c^{2}}+\left(\frac{m^{2} c^{2}}{s-m^{2} c^{2}}+\frac{m^{2} c^{2}}{u-m^{2} c^{2}}\right)^{2}\right. \\
& \left.-\frac{1}{4}\left(\frac{s-m^{2} c^{2}}{u-m^{2} c^{2}}+\frac{u-m^{2} c^{2}}{s-m^{2} c^{2}}\right)\right]
\end{aligned}
$$

is the square of the matrix element, $s=(\mathfrak{p}+\mathfrak{k})^{2}$ and $u=\left(\mathfrak{p}-\mathfrak{k}^{\prime}\right)^{2}$ are invariants, $\mathfrak{k}=$ $\left(\epsilon_{\gamma} / c\right)\left(1, \mathbf{e}_{\gamma}\right)$ and $\mathfrak{p}=\left(\epsilon_{ \pm} / c\right)\left(1, \beta_{ \pm} \mathbf{e}_{ \pm}\right)$are energy-momentum four vectors of photons and electrons, respectively, $d \mathbf{p}=d \epsilon_{ \pm} d o \epsilon_{ \pm}^{2} \beta_{ \pm} / c^{3}, d \mathbf{k}^{\prime}=d \epsilon_{\gamma}^{\prime} \epsilon_{\gamma}^{\prime 2} d o_{\gamma}^{\prime} / c^{3}$ and $d o=d \mu d \phi$. 
The energies of photon and positron (electron) after the scattering are

$$
\epsilon_{\gamma}^{\prime}=\frac{\epsilon_{ \pm} \epsilon_{\gamma}\left(1-\beta_{ \pm} \mathbf{b}_{ \pm} \cdot \mathbf{b}_{\gamma}\right)}{\epsilon_{ \pm}\left(1-\beta_{ \pm} \mathbf{b}_{ \pm} \cdot \mathbf{b}_{\gamma}^{\prime}\right)+\epsilon_{\gamma}\left(1-\mathbf{b}_{\gamma} \cdot \mathbf{b}_{\gamma}^{\prime}\right)}, \quad \epsilon_{ \pm}^{\prime}=\epsilon_{ \pm}+\epsilon_{\gamma}-\epsilon_{\gamma}^{\prime},
$$

$\mathbf{b}_{i}=\mathbf{p}_{i} / p, \mathbf{b}_{i}^{\prime}=\mathbf{p}_{i}^{\prime} / p^{\prime}, \mathbf{b}_{ \pm}^{\prime}=\left(\beta_{ \pm} \epsilon_{ \pm} \mathbf{b}_{ \pm}+\epsilon_{\gamma} \mathbf{b}_{\gamma}-\epsilon_{\gamma}^{\prime} \mathbf{b}_{\gamma}^{\prime}\right) /\left(\beta_{ \pm}^{\prime} \epsilon_{ \pm}^{\prime}\right)$

For photons, the absorption coefficient (10) in the Boltzmann equations (44) is

$$
\chi_{\gamma}^{\gamma e^{ \pm} \rightarrow \gamma^{\prime} e^{ \pm \prime}} f_{\gamma}=-\frac{1}{c}\left(\frac{\partial f_{\gamma}}{\partial t}\right)_{\gamma e^{ \pm} \rightarrow \gamma^{\prime} e^{ \pm \prime}}^{\mathrm{abs}}=\int d n_{ \pm} d o_{\gamma}^{\prime} J_{\mathrm{cs}} \frac{\epsilon_{\gamma}^{\prime}\left|M_{f i}\right|^{2} \hbar^{2} c^{2}}{16 \epsilon_{ \pm} \epsilon_{\gamma} \epsilon_{ \pm}^{\prime}} f_{\gamma}
$$

where $d n_{i}=d \epsilon_{i} d o_{i} \epsilon_{i}^{2} \beta_{i} f_{i} / c^{3}=d \epsilon_{i} d o_{i} E_{i} /\left(2 \pi \epsilon_{i}\right)$.

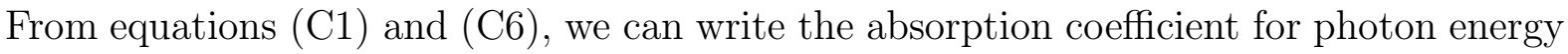
density $E_{\gamma}$ averaged over the $\epsilon, \mu$-grid with zone numbers $\omega$ and $k$ as

$$
\begin{aligned}
(\chi E)_{\gamma, \omega}^{\gamma e^{ \pm} \rightarrow \gamma^{\prime} e^{ \pm \prime}} & \equiv \frac{1}{\Delta \epsilon_{\gamma, \omega}} \int_{\epsilon_{\gamma} \in \Delta \epsilon_{\gamma, \omega}} d \epsilon_{\gamma} d \mu_{\gamma}(\chi E)_{\gamma}^{\gamma e^{ \pm} \rightarrow \gamma^{\prime} e^{ \pm \prime}}= \\
& =\frac{1}{\Delta \epsilon_{\gamma, \omega}} \int_{\epsilon_{\gamma} \in \Delta \epsilon_{\gamma, \omega}} d n_{\gamma} d n_{ \pm} d o_{\gamma}^{\prime} J_{\mathrm{cs}} \frac{\epsilon_{\gamma}^{\prime}\left|M_{f i}\right|^{2} \hbar^{2} c^{2}}{16 \epsilon_{ \pm} \epsilon_{ \pm}^{\prime}}
\end{aligned}
$$

where the Jacobian of the transformation is

$$
J_{\mathrm{cs}}=\frac{\epsilon_{\gamma}^{\prime} \epsilon_{ \pm}^{\prime}}{\epsilon_{\gamma} \epsilon_{ \pm}\left(1-\beta_{ \pm} \mathbf{b}_{\gamma} \cdot \mathbf{b}_{ \pm}\right)}
$$

Similar integrations can be performed for the other terms of equations (C1), (C2), and we obtain

$$
\begin{aligned}
\eta_{\gamma, \omega}^{\gamma e^{ \pm} \rightarrow \gamma^{\prime} e^{ \pm \prime}} & =\frac{1}{\Delta \epsilon_{\gamma, \omega}} \int_{\epsilon_{\gamma}^{\prime} \in \Delta \epsilon_{\gamma, \omega}} d n_{\gamma} d n_{ \pm} d o_{\gamma}^{\prime} J_{\mathrm{cs}} \frac{\epsilon_{\gamma}^{\prime 2}\left|M_{f i}\right|^{2} \hbar^{2} c^{2}}{16 \epsilon_{ \pm} \epsilon_{\gamma} \epsilon_{ \pm}^{\prime}} \\
\eta_{ \pm, \omega}^{\gamma e^{ \pm} \rightarrow \gamma^{\prime} e^{ \pm \prime}} & =\frac{1}{\Delta \epsilon_{ \pm, \omega}} \int_{\epsilon_{ \pm}^{\prime} \in \Delta \epsilon_{ \pm, \omega}} d n_{\gamma} d n_{ \pm} d o_{\gamma}^{\prime} J_{\mathrm{cs}} \frac{\epsilon_{\gamma}^{\prime}\left|M_{f i}\right|^{2} \hbar^{2} c^{2}}{16 \epsilon_{ \pm} \epsilon_{\gamma}} \\
(\chi E)_{ \pm, \omega}^{\gamma e^{ \pm} \rightarrow \gamma^{\prime} e^{ \pm \prime}} & =\frac{1}{\Delta \epsilon_{ \pm, \omega}} \int_{\epsilon_{ \pm} \in \Delta \epsilon_{ \pm, \omega}} d n_{\gamma} d n_{ \pm} d o_{\gamma}^{\prime} J_{\mathrm{cs}} \frac{\epsilon_{\gamma}^{\prime}\left|M_{f i}\right|^{2} \hbar^{2} c^{2}}{16 \epsilon_{\gamma} \epsilon_{ \pm}^{\prime}} .
\end{aligned}
$$

In order to perform integrals (주)-(C11) numerically over $\phi(0 \leq \phi \leq 2 \pi)$ we introduce a uniform grid $\phi_{l \mp 1 / 2}$ with $1 \leq l \leq l_{\max }$ and $\Delta \phi_{l}=\left(\phi_{l+1 / 2}-\phi_{l-1 / 2}\right) / 2=2 \pi / l_{\max }$. We assume that any function of $\phi$ in equations (C7)-(C9) in the interval $\Delta \phi_{j}$ is equal to its value at $\phi=\phi_{j}=\left(\phi_{l-1 / 2}+\phi_{l+1 / 2}\right) / 2$. It is necessary to integrate over $\phi$ only once at the beginning of calculations. The number of intervals of the $\phi$-grid depends on the average energy of particles and is typically taken as $l_{\max }=2 k_{\max }=64$. 


\section{Pair creation and annihilation $\gamma_{1} \gamma_{2} \rightleftarrows e^{-} e^{+}$}

The rates of change of the distribution function due to pair creation and annihilation are

$$
\begin{gathered}
\left(\frac{\partial f_{\gamma_{j}}\left(\mathbf{k}_{i}, t\right)}{\partial t}\right)_{\gamma_{1} \gamma_{2} \rightarrow e^{-} e^{+}}=-\int d \mathbf{k}_{j} d \mathbf{p}_{-} d \mathbf{p}_{+} V w_{\mathbf{p}_{-}, \mathbf{p}_{+} ; \mathbf{k}_{1}, \mathbf{k}_{2}} f_{\gamma_{1}}\left(\mathbf{k}_{1}, t\right) f_{\gamma_{2}}\left(\mathbf{k}_{2}, t\right), \\
\left(\frac{\partial f_{\gamma_{i}}\left(\mathbf{k}_{i}, t\right)}{\partial t}\right)_{e^{-} e^{+\rightarrow \gamma_{1} \gamma_{2}}}=\int d \mathbf{k}_{j} d \mathbf{p}_{-} d \mathbf{p}_{+} V w_{\mathbf{k}_{1}, \mathbf{k}_{2} ; \mathbf{p}_{-}, \mathbf{p}_{+}} f_{-}\left(\mathbf{p}_{-}, t\right) f_{+}\left(\mathbf{p}_{+}, t\right),
\end{gathered}
$$

for $i=1, j=2$, and for $j=1, i=2$.

$$
\begin{gathered}
\left(\frac{\partial f_{ \pm}\left(\mathbf{p}_{ \pm}, t\right)}{\partial t}\right)_{\gamma_{1} \gamma_{2} \rightarrow e^{-} e^{+}}=\int d \mathbf{p}_{\mp} d \mathbf{k}_{1} d \mathbf{k}_{2} V w_{\mathbf{p}_{-}, \mathbf{p}_{+} ; \mathbf{k}_{1}, \mathbf{k}_{2}} f_{\gamma}\left(\mathbf{k}_{1}, t\right) f_{\gamma}\left(\mathbf{k}_{2}, t\right) \\
\left(\frac{\partial f_{ \pm}\left(\mathbf{p}_{ \pm}, t\right)}{\partial t}\right)_{e^{-} e^{+} \rightarrow \gamma_{1} \gamma_{2}}=-\int d \mathbf{p}_{\mp} d \mathbf{k}_{1} d \mathbf{k}_{2} V w_{\mathbf{k}_{1}, \mathbf{k}_{2} ; \mathbf{p}_{-}, \mathbf{p}_{+}} f_{-}\left(\mathbf{p}_{-}, t\right) f_{+}\left(\mathbf{p}_{+}, t\right),
\end{gathered}
$$

where

$$
w_{\mathbf{p}_{-}, \mathbf{p}_{+} ; \mathbf{k}_{1}, \mathbf{k}_{2}}=\frac{\hbar^{2} c^{6}}{(2 \pi)^{2} V} \delta\left(\epsilon_{-}+\epsilon_{+}-\epsilon_{1}-\epsilon_{2}\right) \delta\left(\mathbf{p}_{-}+\mathbf{p}_{+}-\mathbf{k}_{1}-\mathbf{k}_{2}\right) \frac{\left|M_{f i}\right|^{2}}{16 \epsilon_{-} \epsilon_{+} \epsilon_{1} \epsilon_{2}}
$$

Here, the matrix element $\left|M_{f i}\right|^{2}$ is given by equation (C4) with the new invariants $s=$ $\left(\mathfrak{p}_{-}-\mathfrak{k}_{1}\right)^{2}$ and $u=\left(\mathfrak{p}_{-}-\mathfrak{k}_{2}\right)^{2}$, see [41].

The energies of photons created via annihilation of a $e^{ \pm}$pair are

$$
\epsilon_{1}\left(\mathbf{b}_{1}\right)=\frac{m^{2} c^{4}+\epsilon_{-} \epsilon_{+}\left(1-\beta_{-} \beta_{+} \mathbf{b}_{-} \cdot \mathbf{b}_{+}\right)}{\epsilon_{-}\left(1-\beta_{-} \mathbf{b}_{-} \cdot \mathbf{b}_{1}\right)+\epsilon_{+}\left(1-\beta_{+} \mathbf{b}_{+} \cdot \mathbf{b}_{1}\right)}, \quad \epsilon_{2}\left(\mathbf{b}_{1}\right)=\epsilon_{-}+\epsilon_{+}-\epsilon_{1},
$$

while the energies of pair particles created by two photons are found from

$$
\epsilon_{-}\left(\mathbf{b}_{-}\right)=\frac{B \mp \sqrt{B^{2}-A C}}{A}, \quad \epsilon_{+}\left(\mathbf{b}_{-}\right)=\epsilon_{1}+\epsilon_{2}-\epsilon_{-}
$$

where $A=\left(\epsilon_{1}+\epsilon_{2}\right)^{2}-\left[\left(\epsilon_{1} \mathbf{b}_{1}+\epsilon_{2} \mathbf{b}_{2}\right) \cdot \mathbf{b}_{-}\right]^{2}, B=\left(\epsilon_{1}+\epsilon_{2}\right) \epsilon_{1} \epsilon_{2}\left(1-\mathbf{b}_{1} \cdot \mathbf{b}_{2}\right), C=m_{e}^{2} c^{4}\left[\left(\epsilon_{1} \mathbf{b}_{1}+\right.\right.$ $\left.\left.\epsilon_{2} \mathbf{b}_{2}\right) \cdot \mathbf{b}_{-}\right]^{2}+\epsilon_{1}^{2} \epsilon_{2}^{2}\left(1-\mathbf{b}_{1} \cdot \mathbf{b}_{2}\right)^{2}$. Only one root in equation (18) has to be chosen. From energy-momentum conservation

$$
\mathfrak{k}_{1}+\mathfrak{k}_{2}-\mathfrak{p}_{-}=\mathfrak{p}_{+},
$$

taking square from the energy part we have

$$
\epsilon_{1}^{2}+\epsilon_{2}^{2}+\epsilon_{-}^{2}+2 \epsilon_{1} \epsilon_{2}-2 \epsilon_{1} \epsilon_{-}-2 \epsilon_{2} \epsilon_{-}=\epsilon_{+}^{2},
$$


and taking square from the momentum part we get

$$
\epsilon_{1}^{2}+\epsilon_{2}^{2}+\epsilon_{-}^{2} \beta_{-}^{2}+2 \epsilon_{1} \epsilon_{2} \mathbf{b}_{1} \cdot \mathbf{b}_{2}-2 \epsilon_{1} \epsilon_{-} \beta_{-} \mathbf{b}_{1} \cdot \mathbf{b}_{-}-2 \epsilon_{2} \epsilon_{-} \beta_{-} \mathbf{b}_{2} \cdot \mathbf{b}_{-}=\left(\epsilon_{+} \beta_{+}\right)^{2} .
$$

There are no additional roots because of the arbitrary $\mathbf{e}_{+}$

$$
\begin{gathered}
\epsilon_{1} \epsilon_{2}\left(1-\mathbf{b}_{1} \cdot \mathbf{b}_{2}\right)-\epsilon_{1} \epsilon_{-}\left(1-\beta_{-} \mathbf{b}_{1} \cdot \mathbf{b}_{-}\right)-\epsilon_{2} \epsilon_{-}\left(1-\beta \mathbf{b}_{2} \cdot \mathbf{b}_{-}\right)=0 \\
\epsilon_{-} \beta_{-}\left(\epsilon_{1} \mathbf{b}_{1}+\epsilon_{2} \mathbf{b}_{2}\right) \cdot \mathbf{b}_{-}=\epsilon_{-}\left(\epsilon_{1}+\epsilon_{2}\right)-\epsilon_{1} \epsilon_{2}\left(1-\mathbf{b}_{1} \cdot \mathbf{b}_{2}\right)
\end{gathered}
$$

Eliminating $\beta$ we obtain

$$
\begin{aligned}
& \epsilon_{1}^{2} \epsilon_{2}^{2}\left(1-\mathbf{b}_{1} \cdot \mathbf{b}_{2}\right)^{2}-2 \epsilon_{1} \epsilon_{2}\left(1-\mathbf{b}_{1} \cdot \mathbf{b}_{2}\right)\left(\epsilon_{1}+\epsilon_{2}\right) \epsilon_{-}+\left\{\left(\epsilon_{1}+\epsilon_{2}\right)^{2}-\left[\left(\epsilon_{1} \mathbf{b}_{1}+\epsilon_{2} \mathbf{b}_{2}\right) \cdot \mathbf{b}_{-}\right]^{2}\right\} \epsilon_{-}^{2}= \\
& =\left[\left(\epsilon_{1} \mathbf{b}_{1}+\epsilon_{2} \mathbf{b}_{2}\right) \cdot \mathbf{b}_{-}\right]\left(-m^{2}\right),
\end{aligned}
$$

Therefore, the condition to be checked reads

$$
\epsilon_{-} \beta_{-}\left[\left(\epsilon_{1} \mathbf{b}_{1}+\epsilon_{2} \mathbf{b}_{2}\right) \cdot \mathbf{b}_{-}\right]^{2}=\left[\epsilon_{-}\left(\epsilon_{1}+\epsilon_{2}\right)-\left(\epsilon_{1} \epsilon_{2}\right)\left(1-\mathbf{b}_{1} \cdot \mathbf{b}_{2}\right)\right]\left[\left(\epsilon_{1} \mathbf{b}_{1}+\epsilon_{2} \mathbf{b}_{2}\right) \cdot \mathbf{b}_{-}\right] \geq 0
$$

Finally, integration of equations (C12)-(

$$
\begin{aligned}
\eta_{\gamma, \omega}^{e^{-} e^{+} \rightarrow \gamma_{1} \gamma_{2}} & =\frac{1}{\Delta \epsilon_{\gamma, \omega}}\left(\int_{\epsilon_{1} \in \Delta \epsilon_{\gamma, \omega}} d^{2} n_{ \pm} J_{\mathrm{ca}} \frac{\epsilon_{1}^{2}\left|M_{f i}\right|^{2} \hbar^{2} c^{2}}{16 \epsilon_{-} \epsilon_{+} \epsilon_{2}}+\int_{\epsilon_{2} \in \Delta \epsilon_{\gamma, \omega}} d^{2} n_{ \pm} J_{\mathrm{ca}} \frac{\epsilon_{1}\left|M_{f i}\right|^{2} \hbar^{2} c^{2}}{16 \epsilon_{-} \epsilon_{+}}\right) \\
(\chi E)_{e, \omega}^{e^{-} e^{+} \rightarrow \gamma_{1} \gamma_{2}} & =\frac{1}{\Delta \epsilon_{e, \omega}}\left(\int_{\epsilon_{-} \in \Delta \epsilon_{e, \omega}} d^{2} n_{ \pm} J_{\mathrm{ca}} \frac{\epsilon_{1}\left|M_{f i}\right|^{2} \hbar^{2} c^{2}}{16 \epsilon_{+} \epsilon_{2}}+\int_{\epsilon_{+} \in \Delta \epsilon_{e, \omega}} d^{2} n_{ \pm} J_{\mathrm{ca}} \frac{\epsilon_{1}\left|M_{f i}\right|^{2} \hbar^{2} c^{2}}{16 \epsilon_{-} \epsilon_{2}}\right) \\
(\chi E)_{\gamma, \omega}^{\gamma_{1} \gamma_{2} \rightarrow e^{-} e^{+}} & =\frac{1}{\Delta \epsilon_{\gamma, \omega}}\left(\int_{\epsilon_{1} \in \Delta \epsilon_{\gamma, \omega}} d^{2} n_{\gamma} J_{\mathrm{ca}} \frac{\epsilon_{-} \beta_{-}\left|M_{f i}\right|^{2} \hbar^{2} c^{2}}{16 \epsilon_{2} \epsilon_{+}}+\int_{\epsilon_{2} \in \Delta \epsilon_{\gamma, \omega}} d^{2} n_{\gamma} J_{\mathrm{ca}} \frac{\epsilon_{-} \beta_{-}\left|M_{f i}\right|^{2} \hbar^{2} c^{2}}{16 \epsilon_{1} \epsilon_{+}}\right) \\
\eta_{e, \omega}^{\gamma_{1} \gamma_{2} \rightarrow e^{-} e^{+}} & =\frac{1}{\Delta \epsilon_{e, \omega}}\left(\int_{\epsilon_{-} \in \Delta \epsilon_{e, \omega}} d^{2} n_{\gamma} J_{\mathrm{ca}} \frac{\epsilon_{-}^{2} \beta_{-}\left|M_{f i}\right|^{2} \hbar^{2} c^{2}}{16 \epsilon_{1} \epsilon_{2} \epsilon_{+}}+\int_{\epsilon_{+} \in \Delta \epsilon_{e, \omega}} d^{2} n_{\gamma} J_{\mathrm{ca}} \frac{\epsilon_{-} \beta_{-}\left|M_{f i}\right|^{2} \hbar^{2} c^{2}}{16 \epsilon_{1} \epsilon_{2}}\right)
\end{aligned}
$$

where $d^{2} n_{ \pm}=d n_{-} d n_{+} d o_{1}, d^{2} n_{\gamma}=d n_{\gamma_{1}} d n_{\gamma_{2}} d o_{-}, d n_{ \pm}=d \epsilon_{ \pm} d o_{ \pm} \epsilon_{ \pm}^{2} \beta_{ \pm} f_{ \pm}, \quad d n_{\gamma_{1,2}}=$ $d \epsilon_{1,2} d o_{1,2} \epsilon_{1,2}^{2} f_{\gamma_{1,2}}$ and the Jacobian is

$$
J_{\mathrm{ca}}=\frac{\epsilon_{+} \beta_{-}}{\left(\epsilon_{+}+\epsilon_{-}\right) \beta_{-}-\left(\epsilon_{1} \mathbf{b}_{1}+\epsilon_{2} \mathbf{b}_{2}\right) \cdot \mathbf{b}_{-}} .
$$




\section{Møller scattering of electrons and positrons $e_{1}^{ \pm} e_{2}^{ \pm} \rightarrow e_{1}^{ \pm \prime} e_{2}^{ \pm \prime}$}

The time evolution of the distribution functions of electrons (or positrons) is described by

$$
\left(\frac{\partial f_{i}\left(\mathbf{p}_{i}, t\right)}{\partial t}\right)_{e_{1} e_{2} \rightarrow e_{1}^{\prime} e_{2}^{\prime}}=\int d \mathbf{p}_{j} d \mathbf{p}_{1}^{\prime} d \mathbf{p}_{2}^{\prime} V w_{\mathbf{p}_{1}^{\prime}, \mathbf{p}_{2}^{\prime} ; \mathbf{p}_{1}, \mathbf{p}_{2}}\left[f_{1}\left(\mathbf{p}_{1}^{\prime}, t\right) f_{2}\left(\mathbf{p}_{2}^{\prime}, t\right)-f_{1}\left(\mathbf{p}_{1}, t\right) f_{2}\left(\mathbf{p}_{2}, t\right)\right]
$$

with $i=1, j=2$, and with $j=1, i=2$, and where

$$
\begin{aligned}
w_{\mathbf{p}_{1}^{\prime}, \mathbf{p}_{2}^{\prime} ; \mathbf{p}_{1}, \mathbf{p}_{2}} & =\frac{\hbar^{2} c^{6}}{(2 \pi)^{2} V} \delta\left(\epsilon_{1}+\epsilon_{2}-\epsilon_{1}^{\prime}-\epsilon_{2}^{\prime}\right) \delta\left(\mathbf{p}_{1}+\mathbf{p}_{2}-\mathbf{p}_{1}^{\prime}-\mathbf{p}_{2}^{\prime}\right) \frac{\left|M_{f i}\right|^{2}}{16 \epsilon_{1} \epsilon_{2} \epsilon_{1}^{\prime} \epsilon_{2}^{\prime}}, \\
\left|M_{f i}\right|^{2} & =2^{6} \pi^{2} \alpha^{2}\left\{\frac{1}{t^{2}}\left[\frac{s^{2}+u^{2}}{2}+4 m^{2} c^{2}\left(t-m^{2} c^{2}\right)\right]+\right. \\
& \left.+\frac{1}{u^{2}}\left[\frac{s^{2}+t^{2}}{2}+4 m^{2} c^{2}\left(u-m^{2} c^{2}\right)\right]+\frac{4}{t u}\left(\frac{s}{2}-m^{2} c^{2}\right)\left(\frac{s}{2}-3 m^{2} c^{2}\right)\right\},
\end{aligned}
$$

with $s=\left(\mathfrak{p}_{1}+\mathfrak{p}_{2}\right)^{2}=2\left(m^{2} c^{2}+\mathfrak{p}_{1} \mathfrak{p}_{2}\right), t=\left(\mathfrak{p}_{1}-\mathfrak{p}_{1}^{\prime}\right)^{2}=2\left(m^{2} c^{2}-\mathfrak{p}_{1} \mathfrak{p}_{1}^{\prime}\right)$, and $u=\left(\mathfrak{p}_{1}-\mathfrak{p}_{2}^{\prime}\right)^{2}=$ $2\left(m^{2} c^{2}-\mathfrak{p}_{1} \mathfrak{p}_{2}^{\prime}\right)[41]$.

The energies of final-state particles are given by (C18) with new coefficients $\tilde{A}=$ $\left(\epsilon_{1}+\epsilon_{2}\right)^{2}-\left(\epsilon_{1} \beta_{1} \mathbf{b}_{1} \cdot \mathbf{b}_{1}^{\prime}+\epsilon_{2} \beta_{2} \mathbf{b}_{2} \cdot \mathbf{b}_{1}^{\prime}\right)^{2}, \quad \tilde{B}=\left(\epsilon_{1}+\epsilon_{2}\right)\left[m^{2} c^{4}+\epsilon_{1} \epsilon_{2}\left(1-\beta_{1} \beta_{2} \mathbf{b}_{1} \mathbf{b}_{2}\right)\right]$, and $\tilde{C}=m^{2} c^{4}\left(\epsilon_{1} \beta_{1} \mathbf{b}_{1} \cdot \mathbf{b}_{1}^{\prime}+\epsilon_{2} \beta_{2} \mathbf{b}_{2} \cdot \mathbf{b}_{1}^{\prime}\right)^{2}+\left[m^{2} c^{4}+\epsilon_{1} \epsilon_{2}\left(1-\beta_{1} \beta_{2} \mathbf{b}_{1} \cdot \mathbf{b}_{2}\right)\right]^{2}$. The condition to be checked is

$$
\left[\epsilon_{1}^{\prime}\left(\epsilon_{1}+\epsilon_{2}\right)-m^{2} c^{4}-\left(\epsilon_{1} \epsilon_{2}\right)\left(1-\beta_{1} \beta_{2} \mathbf{b}_{1} \cdot \mathbf{b}_{2}\right)\right]\left[\left(\epsilon_{1} \beta_{1} \mathbf{b}_{1}+\epsilon_{2} \beta_{2} \mathbf{b}_{2}\right) \cdot \mathbf{b}_{1}^{\prime}\right] \geq 0
$$

Integration of equations (C30), similar to the case of Compton scattering in Section C1 yields

$$
\begin{aligned}
\eta_{e, \omega}^{e_{1} e_{2} \rightarrow e_{1}^{\prime} e_{2}^{\prime}} & =\frac{1}{\Delta \epsilon_{e, \omega}}\left(\int_{\epsilon_{1}^{\prime} \in \Delta \epsilon_{e, \omega}} d^{2} n J_{\mathrm{ms}} \frac{\epsilon_{1}^{\prime 2} \beta_{1}^{\prime}\left|M_{f i}\right|^{2} \hbar^{2} c^{2}}{16 \epsilon_{1} \epsilon_{2} \epsilon_{2}^{\prime}}+\int_{\epsilon_{2}^{\prime} \in \Delta \epsilon_{e, \omega}} d^{2} n J_{\mathrm{ms}} \frac{\epsilon_{1}^{\prime} \beta_{1}^{\prime}\left|M_{f i}\right|^{2} \hbar^{2} c^{2}}{16 \epsilon_{1} \epsilon_{2}}\right), \\
(\chi E)_{e, \omega}^{e_{1} e_{2} \rightarrow e_{1}^{\prime} e_{2}^{\prime}} & =\frac{1}{\Delta \epsilon_{e, \omega}}\left(\int_{\epsilon_{1} \in \Delta \epsilon_{e, \omega}} d^{2} n J_{\mathrm{ms}} \frac{\epsilon_{1}^{\prime} \beta_{1}^{\prime}\left|M_{f i}\right|^{2} \hbar^{2} c^{2}}{16 \epsilon_{2} \epsilon_{2}^{\prime}}+\int_{\epsilon_{2} \in \Delta \epsilon_{e, \omega}} d^{2} n J_{\mathrm{ms}} \frac{\epsilon_{1}^{\prime} \beta_{1}^{\prime}\left|M_{f i}\right|^{2} \hbar^{2} c^{2}}{16 \epsilon_{1} \epsilon_{2}^{\prime}}\right),
\end{aligned}
$$

where $d^{2} n=d n_{1} d n_{2} d o_{1}^{\prime}, d n_{1,2}=d \epsilon_{1,2} d o_{1,2} \epsilon_{1,2}^{2} \beta_{1,2} f_{1,2}$, and the Jacobian is

$$
J_{\mathrm{ms}}=\frac{\epsilon_{2}^{\prime} \beta_{2}^{\prime}}{\left(\epsilon_{1}^{\prime}+\epsilon_{2}^{\prime}\right) \beta_{1}^{\prime}-\left(\epsilon_{1} \beta_{1} \mathbf{b}_{1}+\epsilon_{2} \beta_{2} \mathbf{b}_{2}\right) \cdot \mathbf{b}_{1}^{\prime}} .
$$




\section{Bhaba scattering of electrons on positrons $e^{-} e^{+} \rightarrow e^{-\prime} e^{+\prime}$}

The time evolution of the distribution functions of electrons and positrons due to Bhaba scattering is described by

$$
\left(\frac{\partial f_{ \pm}\left(\mathbf{p}_{ \pm}, t\right)}{\partial t}\right)_{e^{-} e^{+} \rightarrow e^{-} e^{+\prime}}=\int d \mathbf{p}_{\mp} d \mathbf{p}_{-}^{\prime} d \mathbf{p}_{+}^{\prime} V w_{\mathbf{p}_{-}^{\prime}, \mathbf{p}_{+}^{\prime} ; \mathbf{p}_{-}, \mathbf{p}_{+}}\left[f_{-}\left(\mathbf{p}_{-}^{\prime}, t\right) f_{+}\left(\mathbf{p}_{+}^{\prime}, t\right)-f_{-}\left(\mathbf{p}_{-}, t\right) f_{+}\left(\mathbf{p}_{+}, t\right)\right],
$$

where

$$
w_{\mathbf{p}_{-}^{\prime}, \mathbf{p}_{+}^{\prime} ; \mathbf{p}_{-}, \mathbf{p}_{+}}=\frac{\hbar^{2} c^{6}}{(2 \pi)^{2} V} \delta\left(\epsilon_{-}+\epsilon_{+}-\epsilon_{-}^{\prime}-\epsilon_{+}^{\prime}\right) \delta\left(\mathbf{p}_{-}+\mathbf{p}_{+}-\mathbf{p}_{-}^{\prime}-\mathbf{p}_{+}^{\prime}\right) \frac{\left|M_{f i}\right|^{2}}{16 \epsilon_{-} \epsilon_{+} \epsilon_{-}^{\prime} \epsilon_{+}^{\prime}},
$$

and $\left|M_{f i}\right|$ is given by the equation (C33), but the invariants are $s=\left(\mathfrak{p}_{-}-\mathfrak{p}_{+}^{\prime}\right)^{2}, t=\left(\mathfrak{p}_{+}-\mathfrak{p}_{+}^{\prime}\right)^{2}$ and $u=\left(\mathfrak{p}_{-}+\mathfrak{p}_{+}\right)^{2}$. The final energies $\epsilon_{-}^{\prime}, \epsilon_{+}^{\prime}$ are functions of the outgoing particle directions in a way similar to that in Section C3, see also [41].

Integration of equations (C38) yields

$$
\begin{gathered}
\eta_{ \pm, \omega}^{e^{-} e^{+} \rightarrow e^{-\prime} e^{+\prime}}=\frac{1}{\Delta \epsilon_{ \pm, \omega}}\left(\int_{\epsilon_{-}^{\prime} \in \Delta \epsilon_{e, \omega}} d^{2} n_{ \pm}^{\prime} J_{\mathrm{bs}} \frac{\epsilon_{-}^{\prime 2} \beta_{-}^{\prime}\left|M_{f i}\right|^{2} \hbar^{2} c^{2}}{16 \epsilon_{-} \epsilon_{+} \epsilon_{+}^{\prime}}+\int_{\epsilon_{+}^{\prime} \in \Delta \epsilon_{e, \omega}} d^{2} n_{ \pm}^{\prime} J_{\mathrm{bs}} \frac{\epsilon_{-}^{\prime} \beta_{-}^{\prime}\left|M_{f i}\right|^{2} \hbar^{2} c^{2}}{16 \epsilon_{-} \epsilon_{+}}\right), \\
(\chi E)_{ \pm, \omega}^{e^{-} e^{+} \rightarrow e^{-\prime} e^{+\prime}}=\frac{1}{\Delta \epsilon_{ \pm, \omega}}\left(\int_{\epsilon_{-} \in \Delta \epsilon_{e, \omega}} d^{2} n_{ \pm}^{\prime} J_{\mathrm{bs}} \frac{\epsilon_{-}^{\prime} \beta_{-}^{\prime}\left|M_{f i}\right|^{2} \hbar^{2} c^{2}}{16 \epsilon_{+} \epsilon_{+}^{\prime}}+\int_{\epsilon_{+} \in \Delta \epsilon_{e, \omega}} d^{2} n_{ \pm}^{\prime} J_{\mathrm{bs}} \frac{\epsilon_{-}^{\prime} \beta_{-}^{\prime}\left|M_{f i}\right|^{2} \hbar^{2} c^{2}}{16 \epsilon_{-} \epsilon_{+}^{\prime}}\right),
\end{gathered}
$$

where $d^{2} n_{ \pm}^{\prime}=d n_{-} d n_{+} d o_{-}^{\prime}, d n_{ \pm}=d \epsilon_{ \pm} d o_{ \pm} \epsilon_{ \pm}^{2} \beta_{ \pm} f_{ \pm}$, and the Jacobian is

$$
J_{\mathrm{bs}}=\frac{\epsilon_{+}^{\prime} \beta_{+}^{\prime}}{\left(\epsilon_{-}^{\prime}+\epsilon_{+}^{\prime}\right) \beta_{-}^{\prime}-\left(\epsilon_{-} \beta_{-} \mathbf{b}_{-}+\epsilon_{+} \beta_{+} \mathbf{b}_{+}\right) \cdot \mathbf{b}_{-}^{\prime}} .
$$

Analogously to the case of pair creation and annihilation in Section (C2) the energies of final state particles are given by (지 $)$ with the coefficients $\breve{A}=\left(\epsilon_{-}+\right.$ $\left.\epsilon_{+}\right)^{2}-\left(\epsilon_{-} \beta_{-} \mathbf{b}_{-} \cdot \mathbf{b}_{-}^{\prime}+\epsilon_{+} \beta_{+} \mathbf{b}_{+} \cdot \mathbf{b}_{-}^{\prime}\right)^{2}, \breve{B}=\left(\epsilon_{-}+\epsilon_{+}\right)\left[m^{2} c^{4}+\epsilon_{-} \epsilon_{+}\left(1-\beta_{-} \beta_{+} \mathbf{b}_{-} \cdot \mathbf{b}_{+}\right)\right], \breve{C}=$ $\left[m^{2} c^{4}+\epsilon_{-} \epsilon_{+}\left(1-\beta_{-} \beta_{+} \mathbf{b}_{-} \cdot \mathbf{b}_{+}\right)\right]^{2}+m^{2} c^{4}\left[\epsilon_{-} \beta_{-} \mathbf{b}_{-} \cdot \mathbf{b}_{-}^{\prime}+\epsilon_{+} \beta_{+} \mathbf{b}_{+} \cdot \mathbf{b}_{-}^{\prime}\right]^{2}$. In order to select the correct root one has to check the condition (C34) changing the subscripts $1 \rightarrow-, 2 \rightarrow+$. 


\section{APPENDIX D: BINARY REACTIONS WITH PROTONS}

\section{Compton scattering on protons $\gamma p \rightarrow \gamma^{\prime} p^{\prime}$}

The rate for this process $t_{\gamma p}^{-1}$, compared to the rate of Compton scattering of electrons $t_{\gamma e}^{-1}$ is much longer,

$$
t_{\gamma p}^{-1}=\frac{n_{p}}{n_{ \pm}}\left(\frac{\epsilon_{ \pm}}{M c^{2}}\right)^{2} t_{\gamma e}^{-1} \quad \epsilon \geq m c^{2} .
$$

Moreover, it is longer than any timescale for binary and triple reactions considered in this paper and thus we exclude this reaction from the computations.

\section{Electron-proton and positron-proton scattering $e_{ \pm} p \rightarrow e_{ \pm}^{\prime} p^{\prime}$}

The time evolution of the distribution functions of electrons due to $e p \rightarrow e^{\prime} p^{\prime}$ is described by

$$
\begin{aligned}
& \left(\frac{\partial f_{ \pm}(\mathbf{p}, t)}{\partial t}\right)_{e p \rightarrow e^{\prime} p^{\prime}}=\int d \mathbf{q} d \mathbf{p}^{\prime} d \mathbf{q}^{\prime} V w_{\mathbf{p}^{\prime}, \mathbf{q}^{\prime} ; \mathbf{p}, \mathbf{q}}\left[f_{ \pm}\left(\mathbf{p}^{\prime}, t\right) f_{p}\left(\mathbf{q}^{\prime}, t\right)-f_{ \pm}(\mathbf{p}, t) f_{p}(\mathbf{q}, t)\right] \\
& \left(\frac{\partial f_{p}(\mathbf{q}, t)}{\partial t}\right)_{e p \rightarrow e^{\prime} p^{\prime}}=\int d \mathbf{p} d \mathbf{p}^{\prime} d \mathbf{q}^{\prime} V w_{\mathbf{p}^{\prime}, \mathbf{q}^{\prime} ; \mathbf{p}, \mathbf{q}}\left[f_{ \pm}\left(\mathbf{p}^{\prime}, t\right) f_{p}\left(\mathbf{q}^{\prime}, t\right)-f_{ \pm}(\mathbf{p}, t) f_{p}(\mathbf{q}, t)\right]
\end{aligned}
$$

where

$$
\begin{aligned}
w_{\mathbf{p}^{\prime}, \mathbf{q}^{\prime} ; \mathbf{p}, \mathbf{q}} & =\frac{\hbar^{2} c^{6}}{(2 \pi)^{2} V} \delta\left(\epsilon_{e}+\epsilon_{p}-\epsilon_{e}^{\prime}-\epsilon_{p}^{\prime}\right) \delta\left(\mathbf{p}+\mathbf{q}-\mathbf{p}^{\prime}-\mathbf{q}^{\prime}\right) \frac{\left|M_{f i}\right|^{2}}{16 \epsilon_{e} \epsilon_{p} \epsilon_{e}^{\prime} \epsilon_{p}^{\prime}} \\
\left|M_{f i}\right|^{2} & =2^{6} \pi^{2} \alpha^{2} \frac{1}{t^{2}}\left\{\frac{1}{2}\left(s^{2}+u^{2}\right)+\left(m^{2} c^{2}+M^{2} c^{2}\right)\left(2 t-m^{2} c^{2}-M^{2} c^{2}\right)\right\}
\end{aligned}
$$

the invariants are $s=(\mathfrak{p}+\mathfrak{q})^{2}=m^{2} c^{2}+M^{2} c^{2}+2 \mathfrak{p} \cdot \mathfrak{q}, t=\left(\mathfrak{p}-\mathfrak{p}^{\prime}\right)^{2}=2\left(m^{2} c^{2}-\right.$ $\left.\mathfrak{p} \cdot \mathfrak{p}^{\prime}\right)=2\left(M^{2} c^{2}-\mathfrak{q} \cdot \mathfrak{q}^{\prime}\right)$ and $u=\left(\mathfrak{p}-\mathfrak{q}^{\prime}\right)^{2}=m^{2} c^{2}+M^{2} c^{2}-2 \mathfrak{p} \cdot \mathfrak{q}^{\prime}, s+t+u=$ $2\left(m^{2} c^{2}+M^{2} c^{2}\right)$. The energies of particles after interaction are given by (C18) with $\bar{A}=\left(\epsilon_{ \pm}+\epsilon_{p}\right)^{2}-\left[\left(\epsilon_{ \pm} \beta_{ \pm} \mathbf{b}_{ \pm}+\epsilon_{p} \beta_{p} \mathbf{b}_{p}\right) \cdot \mathbf{b}_{ \pm}^{\prime}\right]^{2}, \bar{B}=\left(\epsilon_{ \pm}+\epsilon_{p}\right)\left[m^{2} c^{4}+\epsilon_{ \pm} \epsilon_{p}\left(1-\beta_{ \pm} \beta_{p} \mathbf{b}_{ \pm} \cdot \mathbf{b}_{p}\right)\right]$,

$\bar{C}=m^{2} c^{4}\left\{\left(\epsilon_{ \pm} \beta_{ \pm} \mathbf{b}_{ \pm} \cdot \mathbf{b}_{ \pm}^{\prime}+\epsilon_{p} \beta_{p} \mathbf{b}_{p} \cdot \mathbf{b}_{ \pm}^{\prime}\right)^{2}+\left[m^{2} c^{4}+\epsilon_{ \pm} \epsilon_{p}\left(1-\beta_{ \pm} \beta_{p} \mathbf{b}_{ \pm} \cdot \mathbf{b}_{p}\right)\right]\right\}^{2}$. The correct root is selected by the condition (C34) with the substitution $1 \rightarrow \pm, 2 \rightarrow p$. 
Absorption and emission coefficients for this reaction are

$$
\begin{aligned}
(\chi E)_{ \pm, \omega}^{e p} & =\frac{1}{\Delta \epsilon_{ \pm, \omega}} \int_{\epsilon_{ \pm} \in \Delta \epsilon_{ \pm, \omega}} d n_{ \pm} d n_{p} d o_{ \pm}^{\prime} J_{\mathrm{ep}} \frac{\epsilon_{ \pm}^{\prime 2} \beta_{ \pm}^{\prime} \epsilon_{ \pm}\left|M_{f i}\right|^{2} \hbar^{2} c^{2}}{16 \epsilon_{ \pm} \epsilon_{p} \epsilon_{ \pm}^{\prime} \epsilon_{p}^{\prime}} \\
(\chi E)_{p, \omega}^{e p} & =\frac{1}{\Delta \epsilon_{p, \omega}} \int_{\epsilon_{p} \in \Delta \epsilon_{p, \omega}} d n_{ \pm} d n_{p} d o_{ \pm}^{\prime} J_{\mathrm{ep}} \frac{\epsilon_{ \pm}^{\prime 2} \beta_{ \pm}^{\prime} \epsilon_{p}\left|M_{f i}\right|^{2} \hbar^{2} c^{2}}{16 \epsilon_{ \pm} \epsilon_{p} \epsilon_{ \pm}^{\prime} \epsilon_{p}^{\prime}} \\
\eta_{ \pm, \omega}^{e p} & =\frac{1}{\Delta \epsilon_{ \pm, \omega}} \int_{\epsilon_{ \pm}^{\prime} \in \Delta \epsilon_{ \pm, \omega}} d n_{ \pm} d n_{p} d o_{ \pm}^{\prime} J_{\mathrm{ep}} \frac{\epsilon_{ \pm}^{\prime 2} \beta_{ \pm}^{\prime} \epsilon_{ \pm}^{\prime}\left|M_{f i}\right|^{2} \hbar^{2} c^{2}}{16 \epsilon_{ \pm} \epsilon_{p} \epsilon_{ \pm}^{\prime} \epsilon_{p}^{\prime}} \\
\eta_{p, \omega}^{e p} & =\frac{1}{\Delta \epsilon_{p, \omega}} \int_{\epsilon_{p}^{\prime} \in \Delta \epsilon_{p, \omega}} d n_{ \pm} d n_{p} d o_{ \pm}^{\prime} J_{\mathrm{ep}} \frac{\epsilon_{ \pm}^{\prime 2} \beta_{ \pm}^{\prime} \epsilon_{p}^{\prime}\left|M_{f i}\right|^{2} \hbar^{2} c^{2}}{16 \epsilon_{ \pm} \epsilon_{p} \epsilon_{ \pm}^{\prime} \epsilon_{p}^{\prime}}
\end{aligned}
$$

where $d n_{i}=d \epsilon_{i} d o_{i} \epsilon_{i}^{2} \beta_{i} f_{i}, i= \pm, p$, and the Jacobian is

$$
J_{\mathrm{ep}}=\frac{\epsilon_{p}^{\prime} \beta_{p}^{\prime}}{\left(\epsilon_{ \pm}^{\prime}+\epsilon_{p}^{\prime}\right) \beta_{ \pm}^{\prime}-\left(\epsilon_{p} \beta_{p} \mathbf{b}_{p}+\epsilon_{ \pm} \beta_{ \pm} \mathbf{b}_{ \pm}\right) \cdot \mathbf{b}_{ \pm}^{\prime}} .
$$

The rate for proton-electron (proton-positron) scattering is

$$
t_{e p}^{-1} \approx \frac{\epsilon}{M c^{2}} t_{e e}^{-1}, \quad \epsilon_{ \pm} \ll \epsilon_{p}
$$

\section{Proton-proton scattering $p_{1} p_{2} \rightarrow p_{1}^{\prime} p_{2}^{\prime}$}

This reaction is similar to $e_{1} e_{2} \rightarrow e_{1}^{\prime} e_{2}^{\prime}$, described in Section C3. The time evolution of the distribution functions of electrons is described by

$$
\left(\frac{\partial f_{i}\left(\mathbf{p}_{i}, t\right)}{\partial t}\right)_{p_{1} p_{2} \rightarrow p_{1}^{\prime} p_{2}^{\prime}}=\int d \mathbf{q}_{j} d \mathbf{q}_{1}^{\prime} d \mathbf{q}_{2}^{\prime} V w_{\mathbf{q}_{1}^{\prime}, \mathbf{q}_{2}^{\prime} ; \mathbf{q}_{1}, \mathbf{q}_{2}}\left[f_{1}\left(\mathbf{q}_{1}^{\prime}, t\right) f_{2}\left(\mathbf{q}_{2}^{\prime}, t\right)-f_{1}\left(\mathbf{q}_{1}, t\right) f_{2}\left(\mathbf{q}_{2}, t\right)\right]
$$

with $j=3-i$, and where

$$
\begin{aligned}
& w_{\mathbf{q}_{1}^{\prime}, \mathbf{q}_{2}^{\prime} ; \mathbf{q}_{1}, \mathbf{q}_{2}}=\frac{\hbar^{2} c^{6}}{(2 \pi)^{2} V} \delta\left(\epsilon_{1}+\epsilon_{2}-\epsilon_{1}^{\prime}-\epsilon_{2}^{\prime}\right) \delta\left(\mathbf{q}_{1}+\mathbf{q}_{2}-\mathbf{q}_{1}^{\prime}-\mathbf{q}_{2}^{\prime}\right) \frac{\left|M_{f i}\right|^{2}}{16 \epsilon_{1} \epsilon_{2} \epsilon_{1}^{\prime} \epsilon_{2}^{\prime}}, \\
&\left|M_{f i}\right|^{2}=2^{6} \pi^{2} \alpha^{2}\left\{\frac{1}{t^{2}}\left[\frac{s^{2}+u^{2}}{2}+4 M^{2} c^{2}\left(t-M^{2} c^{2}\right)\right]+\right. \\
&\left.\frac{1}{u^{2}}\left[\frac{s^{2}+t^{2}}{2}+4 M^{2} c^{2}\left(u-M^{2} c^{2}\right)\right]+\frac{4}{t u}\left(\frac{s}{2}-M^{2} c^{2}\right)\left(\frac{s}{2}-3 M^{2} c^{2}\right)\right\},
\end{aligned}
$$

and the invariants are $s=\left(\mathfrak{q}_{1}+\mathfrak{q}_{2}\right)^{2}=2\left(M^{2} c^{2}+\mathfrak{q}_{1} \cdot \mathfrak{q}_{2}\right), t=\left(\mathfrak{q}_{1}-\mathfrak{q}_{1}^{\prime}\right)^{2}=2\left(M^{2} c^{2}-\mathfrak{q}_{1} \cdot \mathfrak{q}_{1}^{\prime}\right)$, and $u=\left(\mathfrak{q}_{1}-\mathfrak{q}_{2}^{\prime}\right)^{2}=2\left(M^{2} c^{2}-\mathfrak{q}_{1} \mathfrak{q}_{2}^{\prime}\right)$.

For the rate we have

$$
t_{p p}^{-1} \approx \sqrt{\frac{m}{M}} \frac{n_{p}}{n_{ \pm}} t_{e e}^{-1}, \quad v_{p} \approx \sqrt{\frac{m}{M}} v_{ \pm}, \quad v_{ \pm} \approx c
$$




\section{APPENDIX E: THREE-BODY PROCESSES}

We adopt emission coefficients for triple interactions from [31].

Bremsstrahlung

$$
\begin{gathered}
\eta_{\gamma}^{e^{\mp} e^{\mp} \rightarrow e^{\mp} e^{\mp} \gamma}=\left(n_{+}^{2}+n_{-}^{2}\right) \frac{16}{3} \frac{\alpha c}{\varepsilon}\left(\frac{e^{2}}{m c^{2}}\right)^{2} \ln \left[4 \xi\left(11.2+10.4 \theta^{2}\right) \frac{\theta}{\varepsilon}\right] \frac{\frac{3}{5} \sqrt{2} \theta+2 \theta^{2}}{\exp (1 / \theta) K_{2}(1 / \theta)}, \\
\eta_{\gamma}^{e^{-} e^{+} \rightarrow e^{-} e^{+} \gamma}=n_{+} n_{-} \frac{16}{3} \frac{2 \alpha c}{\varepsilon}\left(\frac{e^{2}}{m c^{2}}\right)^{2} \ln \left[4 \xi\left(1+10.4 \theta^{2}\right) \frac{\theta}{\varepsilon}\right] \frac{\sqrt{2}+2 \theta+2 \theta^{2}}{\exp (1 / \theta) K_{2}(1 / \theta)} \\
\eta_{\gamma}^{p e^{ \pm} \rightarrow p^{\prime} e^{ \pm \prime} \gamma}=\left(n_{+}+n_{-}\right) n_{p} \frac{16}{3} \frac{\alpha c}{\varepsilon}\left(\frac{e^{2}}{m c^{2}}\right)^{2} \ln \left[4 \xi(1+3.42 \theta) \frac{\theta}{\varepsilon}\right] \frac{1+2 \theta+2 \theta^{2}}{\exp (1 / \theta) K_{2}(1 / \theta)}
\end{gathered}
$$

where $\xi=e^{-0.5772}$, and $K_{2}(1 / \theta)$ is the modified Bessel function of the second kind of order 2.

Double Compton scattering

$$
\eta_{\gamma}^{e^{ \pm} \gamma \rightarrow e^{ \pm \prime} \gamma^{\prime} \gamma^{\prime \prime}}=\left(n_{+}+n_{-}\right) n_{\gamma} \frac{128}{3} \frac{\alpha c}{\varepsilon}\left(\frac{e^{2}}{m c^{2}}\right)^{2} \frac{\theta^{2}}{1+13.91 \theta+11.05 \theta^{2}+19.92 \theta^{3}},
$$

Three photon annihilation

$$
\eta_{\gamma}^{e^{ \pm} e^{\mp} \rightarrow \gamma \gamma^{\prime} \gamma^{\prime \prime}}=n_{+} n_{-} \alpha c\left(\frac{e^{2}}{m c^{2}}\right)^{2} \frac{1}{\varepsilon} \frac{\frac{4}{\theta}\left(2 \ln ^{2} 2 \xi \theta+\frac{\pi^{2}}{6}-\frac{1}{2}\right)}{4 \theta+\frac{1}{\theta^{2}}\left(2 \ln ^{2} 2 \xi \theta+\frac{\pi^{2}}{6}-\frac{1}{2}\right)},
$$

where we have joined two limiting approximations given by [31].

Radiative pair production

$$
\eta_{e}^{\gamma \gamma^{\prime} \rightarrow \gamma^{\prime \prime} e^{ \pm} e^{\mp}}=\eta_{\gamma}^{e^{ \pm} e^{\mp} \rightarrow \gamma \gamma^{\prime} \gamma^{\prime \prime}} \frac{n_{\gamma}^{2}}{n_{+} n_{-}}\left[\frac{K_{2}(1 / \theta)}{2 \theta^{2}}\right]^{2} .
$$

Electron-photon pair production

$$
\eta_{\gamma}^{e_{1}^{ \pm} \gamma \rightarrow e_{1}^{ \pm \prime} e^{ \pm} e^{\mp}}=\left\{\begin{array}{cc}
\left(n_{+}+n_{-}\right) n_{\gamma} \alpha c\left(\frac{e^{2}}{m c^{2}}\right)^{2} \exp \left(-\frac{2}{\theta}\right) 16.1 \theta^{0.541}, & \theta \leq 2, \\
\left(n_{+}+n_{-}\right) n_{\gamma} \alpha c\left(\frac{e^{2}}{m c^{2}}\right)^{2}\left(\frac{56}{9} \ln 2 \xi \theta-\frac{8}{27}\right) \frac{1}{1+0.5 / \theta}, & \theta>2 .
\end{array}\right.
$$

Proton-photon pair production

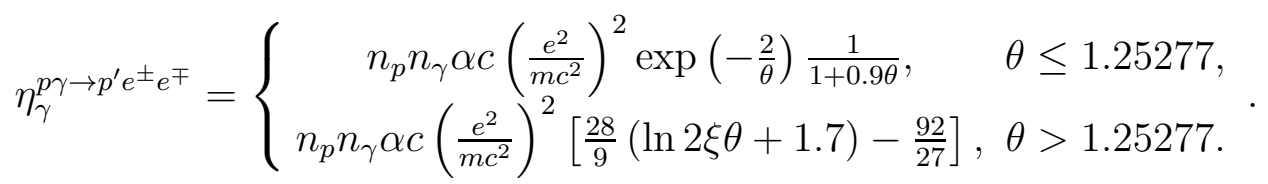

We use the absorption coefficient for three-body processes written as

$$
\chi_{\gamma}^{3 \mathrm{p}}=\eta_{\gamma}^{3 \mathrm{p}} / E_{\gamma}^{\mathrm{eq}}
$$


where $\eta_{\gamma}^{3 \mathrm{p}}$ is the sum of the emission coefficients of photons in the three particle processes, $E_{\gamma}^{\mathrm{eq}}=2 \pi \epsilon^{3} f_{\gamma}^{\mathrm{eq}} / c^{3}$, where $f_{\gamma}^{\mathrm{eq}}$ is given by (15) .

From equation (22), the law of energy conservation in the three-body processes is

$$
\int \sum_{i}\left(\eta_{i}^{3 \mathrm{p}}-\chi_{i}^{3 \mathrm{p}} E_{i}\right) d \mu d \epsilon=0
$$

For exact conservation of energy in these processes we introduce the following coefficients of emission and absorption for electrons:

$$
\chi_{e}^{3 \mathrm{p}}=\frac{\int\left(\eta_{\gamma}^{3 \mathrm{p}}-\chi_{\gamma}^{3 \mathrm{p}} E_{\gamma}\right) d \epsilon d \mu}{\int E_{e} d \epsilon d \mu}, \quad \eta_{e}^{3 \mathrm{p}}=0, \quad \int\left(\eta_{\gamma}^{3 \mathrm{p}}-\chi_{\gamma}^{3 \mathrm{p}} E_{\gamma}\right) d \epsilon d \mu>0,
$$

or

$$
\frac{\eta_{e}^{3 \mathrm{p}}}{E_{e}}=-\frac{\int\left(\eta_{\gamma}^{3 \mathrm{p}}-\chi_{\gamma}^{3 \mathrm{p}} E_{\gamma}\right) d \epsilon d \mu}{\int E_{e} d \epsilon d \mu}, \quad \chi_{e}^{3 \mathrm{p}}=0, \quad \int\left(\eta_{\gamma}^{3 \mathrm{p}}-\chi_{\gamma}^{3 \mathrm{p}} E_{\gamma}\right) d \epsilon d \mu<0
$$

\section{APPENDIX F: CUTOFF IN THE COULOMB SCATTERING}

Denote quantities in the center of mass (CM) frame with index 0 , and with prime after interaction. Suppose we have two particles with masses $m_{1}$ and $m_{2}$. The change of the angle of the first particle in CM system is

$$
\theta_{10}=\arccos \left(\mathbf{b}_{10} \cdot \mathbf{b}_{10}^{\prime}\right)
$$

the numerical grid size is $\Delta \theta_{\mathrm{g}}$, the minimal angle at the scattering is $\theta_{\min }$.

By definition in the in CM frame

$$
\mathbf{p}_{10}+\mathbf{p}_{20}=0
$$

where

$$
\mathbf{p}_{i 0}=\mathbf{p}_{i}+\left[(\Gamma-1)\left(\mathbf{N p}_{i}\right)-\Gamma \frac{V}{c} \frac{\epsilon_{i}}{c}\right] \mathbf{N}, \quad i=1,2
$$

and

$$
\epsilon_{i}=\Gamma\left(\epsilon_{i 0}+\mathbf{V} \mathbf{p}_{i 0}\right)
$$

Then for the velocity of the CM frame we have

$$
\frac{\mathbf{V}}{c}=c \frac{\mathbf{p}_{1}+\mathbf{p}_{2}}{\epsilon_{1}+\epsilon_{2}}, \quad \mathbf{N}=\frac{\mathbf{V}}{V}, \quad \Gamma=\frac{1}{\sqrt{1-\left(\frac{V}{c}\right)^{2}}} .
$$


By definition

$$
\mathbf{b}_{10}=\mathbf{b}_{20}, \quad \mathbf{b}_{10}^{\prime}=\mathbf{b}_{20}^{\prime},
$$

and then

$$
\begin{gathered}
\left|\mathbf{p}_{10}\right|=\left|\mathbf{p}_{20}\right|=p_{0} \equiv \\
\equiv \frac{1}{c} \sqrt{\epsilon_{10}^{2}-m_{1}^{2} c^{4}}=\frac{1}{c} \sqrt{\epsilon_{20}^{2}-m_{2}^{2} c^{4}}
\end{gathered}
$$

where

$$
\begin{aligned}
& \epsilon_{10}=\frac{\left(\epsilon_{1}+\epsilon_{2}\right)^{2}-\Gamma^{2}\left(m_{2}^{2}-m_{1}^{2}\right) c^{4}}{2\left(\epsilon_{1}+\epsilon_{2}\right) \Gamma}, \\
& \epsilon_{20}=\frac{\left(\epsilon_{1}+\epsilon_{2}\right)^{2}+\Gamma^{2}\left(m_{2}^{2}-m_{1}^{2}\right) c^{4}}{2\left(\epsilon_{1}+\epsilon_{2}\right) \Gamma} .
\end{aligned}
$$

Haug [44] gives the minimal scattering angle in the center of mass system

$$
\theta_{\min }=\frac{2 \hbar}{\mathcal{M} c D} \frac{\gamma_{r}}{\left(\gamma_{r}+1\right) \sqrt{2\left(\gamma_{r}-1\right)}}
$$

where $\mathcal{M}$, as above, is the reduced mass, the maximum impact parameter (neglecting the effect of protons) is

$$
D=\frac{c^{2}}{\omega} \frac{p_{0}}{\epsilon_{10}}
$$

and the invariant Lorentz factor of relative motion (e.g. [44]) is

$$
\gamma_{r}=\frac{1}{\sqrt{1-\left(\frac{v_{r}}{c}\right)^{2}}}=\frac{\epsilon_{1} \epsilon_{2}-\mathbf{p}_{1} \mathbf{p}_{2} c^{2}}{m_{1} m_{2} c^{4}} .
$$

In the CM frame we finally obtain

$$
t_{\text {min }}=2\left[(m c)^{2}-\left(\frac{\epsilon_{10}}{c}\right)^{2}\left(1-\beta_{10}^{2} \cos \theta_{\text {min }}\right)\right]
$$

Since it is invariant, we then replace $t$ in the denominator of $\left|M_{f i}\right|^{2}$ in (C33) by the value $t \sqrt{1+t_{\min }^{2} / t^{2}}$ to implement the cutoff scheme. Also at the scattering of equivalent particles we remove the case of exchange of particles as well as scattering on small angles, in other words we change $u$ in the denominator of $\left|M_{f i}\right|^{2}$ in (ㅈ3), (D5) and (D14) by the value $u \sqrt{1+t_{\min }^{2} / u^{2}}$. 
APPENDIX G: MASS SCALING FOR THE PROTON-ELECTRON/POSITRON REACTION

Since proton mass is larger than electron mass-energy $M \gg m, \epsilon$ then for the CM frame

$$
\begin{aligned}
& \mathbf{V} \approx \frac{\mathbf{p}_{1}+\mathbf{p}_{2}}{M}, \quad \Gamma \approx 1, \quad J_{1} \approx 1 \\
& \epsilon_{1}^{\prime}-\epsilon_{1} \approx \mathbf{V}\left(\mathbf{e}_{01}^{\prime}-\mathbf{e}_{01}\right) p_{0} \propto \frac{1}{M}
\end{aligned}
$$

and also

$$
\begin{gathered}
\frac{s^{2}}{c^{4}} \approx M^{4}+4 m M^{3}+6 m^{2} M^{2}, \\
\frac{u^{2}}{c^{4}} \approx M^{4}-4 m M^{3}+6 m^{2} M^{2}, \\
\left|M_{f i}\right|^{2} \propto \frac{1}{t^{2}}\left(6 m^{2}-2 t\right) M^{2},
\end{gathered}
$$

while

$$
\begin{aligned}
t & =\frac{-2 m^{2} \beta_{e 0}^{2}\left(1-\mathbf{e}_{e 0} \mathbf{e}_{e 0}^{\prime}\right)}{1-\beta_{e 0}^{2}}= \\
& =\frac{-2 m^{2} \beta_{e}^{2}\left(1-\mathbf{e}_{e} \mathbf{e}_{e}^{\prime}\right)}{1-\beta_{e}^{2}}\left[1+O\left(M^{-1}\right)\right]
\end{aligned}
$$

for small angles.

This leads to the following scaling for the reaction rate

$$
\eta_{e \omega}^{e p}-(\chi E)_{e \omega}^{e p} \propto \int \frac{\left(\epsilon_{e}^{\prime}-\epsilon_{e}\right)\left|M_{f i}\right|^{2}}{\epsilon_{e} \epsilon_{p} \epsilon_{e}^{\prime} \epsilon_{p}^{\prime}} \propto \frac{1}{M}
$$

We can therefore calculate $\eta_{e \omega}^{e p_{0}},(\chi E)_{e \omega}^{e p_{0}}$ for a pseudo-particle with mass $M_{0} \gg m, \epsilon$ instead of $M$ and obtain

$$
\begin{aligned}
\eta_{e \omega}^{e p} & \approx \frac{M_{0}}{M} \eta_{e \omega}^{e p_{0}}, \\
(\chi E)_{e \omega}^{e p} & \approx \frac{M_{0}}{M}(\chi E)_{e \omega}^{e p_{0}} .
\end{aligned}
$$

For such purpose we selected the mass of this pseudo-particle as $M_{0}=20 \mathrm{~m}$. 


\section{APPENDIX H: THE DEFINITION OF MATRIX ELEMENTS}

Following [41] define the scattering matrix, being composed of real and imaginary parts

$$
S_{f i}=\delta_{f i}+i(2 \pi \hbar)^{4} \delta^{(4)}\left(\mathfrak{p}_{f}-\mathfrak{p}_{i}\right) T_{f i}
$$

where $\delta_{f i}$ is the unity matrix, $\delta^{(4)}$ stands for the four-momentum conservation and the elements of $T_{f i}$ are scattering amplitudes.

The transition probability of a given process per unit time is then

$$
w_{f i}=c(2 \pi \hbar)^{4} \delta^{(4)}\left(\mathfrak{p}_{f}-\mathfrak{p}_{i}\right)\left|T_{f i}\right|^{2} V
$$

where $V$ is the normalization volume.

For a process involving $a$ outgoing particles and $b$ incoming particles the differential probability per unit time is defined as

$$
\begin{aligned}
d w & =c(2 \pi \hbar)^{4} \delta^{(4)}\left(\mathfrak{p}_{f}-\mathfrak{p}_{i}\right)\left|M_{f i}\right|^{2} V \times \\
& \times\left[\prod_{b} \frac{\hbar c}{2 \epsilon_{b} V}\right]\left[\prod_{a} \frac{d \mathbf{p}_{a}^{\prime}}{(2 \pi \hbar)^{3}} \frac{\hbar c}{2 \epsilon_{a}^{\prime}}\right],
\end{aligned}
$$

where $\mathbf{p}_{a}^{\prime}$ and $\epsilon_{a}^{\prime}$ are respectively momenta and energies of outgoing particles, $\epsilon_{b}$ are energies of particles before interaction, $M_{f i}$ are the corresponding matrix elements, $\delta^{(4)}$ stands for energy-momentum conservation, $V$ is the normalization volume. The matrix elements are related to the scattering amplitudes by

$$
M_{f i}=\left[\prod_{b} \frac{\hbar c}{2 \epsilon_{b} V}\right]\left[\prod_{a} \frac{\hbar c}{2 \epsilon_{a}^{\prime} V}\right] T_{f i} .
$$

For a binary process with 2 incoming and 2 outgoing particles it is convenient to introduce the differential cross-section. In fact, the differential probability for incoming particles with four momenta $\mathfrak{p}_{1}$ and $\mathfrak{p}_{2}$, energies $\epsilon_{1}$ and $\epsilon_{2}$ and masses $m_{1}$ and $m_{2}$ respectively, is just the product of the differential cross-section and the flux density

$$
d w=j d \sigma
$$

where

$$
\begin{aligned}
j & =\frac{c I}{\epsilon_{1} \epsilon_{2} V}, \\
I & =c \sqrt{\mathfrak{p}_{1} \mathfrak{p}_{2}-m_{1} m_{2} c^{2}} .
\end{aligned}
$$


In the CM reference frame the relation between the cross section and $\left|M_{f i}\right|^{2}$ acquires simplest form if cross-section is independent on the azimuth of $\mathbf{p}_{1}^{\prime}$ relative to $\mathbf{p}_{1}$ then

$$
\begin{aligned}
d \sigma & =\frac{\hbar^{2} c^{4}}{64 \pi}\left|M_{f i}\right|^{2} \frac{d t}{I}, \\
t & =\left(\mathfrak{p}_{1}-\mathfrak{p}_{2}\right)^{2}, \\
d t & =2\left|\mathbf{p}_{1}\right|\left|\mathbf{p}_{1}^{\prime}\right| d \cos \vartheta
\end{aligned}
$$

where $\vartheta$ is the angle between $\mathbf{p}_{1}$ and $\mathbf{p}_{1}^{\prime}$.

[1] A. G. Aksenov, R. Ruffini, and G. V. Vereshchagin, Phys. Rev. Lett.99, 125003 (2007).

[2] S. Weinberg, Gravitation and Cosmology: Principles and Applications of the General Theory of Relativity, Gravitation and Cosmology: Principles and Applications of the General Theory of Relativity, by Steven Weinberg, pp. 688. ISBN 0-471-92567-5. Wiley-VCH , July 1972., 1972.

[3] E. W. Kolb and M. S. Turner, The Early Universe, Frontiers in Physics, Reading, MA: Addison-Wesley, 1990.

[4] W. T. Hu, Wandering in the Background: a Cosmic Microwave Background Explorer, $\mathrm{PhD}$ thesis, AA(UNIVERSITY OF CALIFORNIA, BERKELEY.), 1995.

[5] S. Weinberg, Cosmology, Oxford University Press, April 2008., 2008.

[6] J. Goodman, ApJ 308, L47 (1986).

[7] T. Piran, Phys. Rep. 314, 575 (1999).

[8] R. Ruffini, J. D. Salmonson, J. R. Wilson, and S.-S. Xue, A\&A 350, 334 (1999).

[9] J. F. C. Wardle, D. C. Homan, R. Ojha, and D. H. Roberts, Nature395, 457 (1998).

[10] E. Churazov, R. Sunyaev, S. Sazonov, M. Revnivtsev, and D. Varshalovich, MNRAS 357, 1377 (2005).

[11] V. V. Usov, Phys. Rev. Lett.80, 230 (1998).

[12] D. B. Blaschke, A. V. Prozorkevich, C. D. Roberts, S. M. Schmidt, and S. A. Smolyansky, Phys. Rev. Lett.96, 140402 (2006).

[13] I. Kuznetsova, D. Habs, and J. Rafelski, Physical Review D (Particles and Fields) 78, 014027 (2008).

[14] G. S. Bisnovatyi-Kogan, Y. B. Zel'Dovich, and R. A. Syunyaev, Soviet Ast. 15, 17 (1971). 
[15] T. A. Weaver, Phys. Rev. A13, 1563 (1976).

[16] A. P. Lightman, ApJ 253, 842 (1982).

[17] R. J. Gould, ApJ 254, 755 (1982).

[18] S. Stepney and P. W. Guilbert, MNRAS 204, 1269 (1983).

[19] P. S. Coppi and R. D. Blandford, MNRAS 245, 453 (1990).

[20] A. P. Lightman and D. L. Band, ApJ 251, 713 (1981).

[21] R. Svensson, ApJ 258, 335 (1982).

[22] P. W. Guilbert and S. Stepney, MNRAS 212, 523 (1985).

[23] A. A. Zdziarski, ApJ 283, 842 (1984).

[24] R. J. Gould, Physics of Fluids 24, 102 (1981).

[25] S. Stepney, MNRAS 202, 467 (1983).

[26] R. Svensson, ApJ 258, 321 (1982).

[27] R. J. Gould, ApJ 238, 1026 (1980).

[28] E. Haug, A\&A 148, 386 (1985).

[29] A. P. Lightman, ApJ 244, 392 (1981).

[30] R. J. Gould, ApJ 285, 275 (1984).

[31] R. Svensson, MNRAS 209, 175 (1984).

[32] S. Iwamoto and F. Takahara, ApJ 601, 78 (2004).

[33] G. Cavallo and M. J. Rees, MNRAS 183, 359 (1978).

[34] L. D. Landau and E. M. Lifshitz, Physical Kinetics, Elsevier, 1981.

[35] A. G. Aksenov, R. Ruffini, and G. V. Vereshchagin, Thermalization of Electron-PositronPhoton Plasmas with an application to GRB, in American Institute of Physics Conference Series, volume 966 of American Institute of Physics Conference Series, pages 191-196, 2008.

[36] R. Ruffini et al., The Blackholic energy and the canonical Gamma-Ray Burst, in American Institute of Physics Conference Series, volume 910 of American Institute of Physics Conference Series, pages 55-217, 2007.

[37] S. Belyaev and G. Budker, DAN SSSR 107, 807 (1956).

[38] D. Mihalas and B. W. Mihalas, Foundations of Radiation Hydrodynamics, New York, Oxford University Press, 1984.

[39] A. G. Aksenov, M. Milgrom, and V. V. Usov, ApJ 609, 363 (2004).

[40] D. Lemoine, Phys. Rev. D 51, 2677 (1995). 
[41] V. B. Berestetskii, E. M. Lifshitz, and V. B. Pitaevskii, Quantum Electrodynamics, Elsevier, 1982.

[42] W. Greiner and J. Reinhardt, Quantum Electrodynamics, Berlin, Springer, 2003.

[43] A. Akhiezer and V. Berestetskii, Quantum Electrodynamics, Moscow, Nauka, 1981.

[44] E. Haug, A\&A 191, 181 (1988).

[45] R. P. Pilla and J. Shaham, ApJ 486, 903 (1997).

[46] J. Ehlers, Survey of general relativity theory, in Relativity, Astrophysics and Cosmology, pages $1-125,1973$.

[47] G. Hall and J. M. Watt, Modern Numerical Methods for Ordinary Differential Equations, New York, Oxford University Press, 1976.

[48] R. Ruffini, J. D. Salmonson, J. R. Wilson, and S.-S. Xue, A\&A 359, 855 (2000).

[49] R. Ruffini, G. Vereshchagin, and S.-S. Xue, Physics Reports , in press (2009).

[50] M. H. Thoma, ArXiv e-prints (2008).

[51] N. Chernikov, Acta Physica Polonica 23, 629 (1963).

[52] I. P. Ochelkov, O. F. Prilutskii, I. L. Rozental, and V. V. Usov, Relativistic kinetics and hydrodynamics, Moscow, Atomizdat, 1979. 